Supporting Information

\title{
Enhanced Sulfate Production by Nitrate Photolysis in the Presence of Halide Ions in Atmospheric Particles
}

Ruifeng Zhang ${ }^{1}$, Masao Gen ${ }^{1,2 *}$, Dandan Huang ${ }^{3}$, Yongjie $\mathrm{Li}^{4}$, Chak K. Chan ${ }^{1 *}$

${ }^{1}$ School of Energy and Environment, City University of Hong Kong, Tat Chee Avenue, Kowloon, Hong Kong, China

${ }^{2}$ Faculty of Frontier Engineering, Institute of Science and Engineering, Kanazawa University, Kakuma-machi, Kanazawa 920-1192, Japan

${ }^{3}$ Shanghai Academy of Environmental Sciences, Shanghai 200233, China

${ }^{4}$ Department of Civil and Environmental Engineering, Faculty of Science and Technology, University of Macau, Macau 999078, China

\section{Corresponding Author}

*Author to whom correspondence should be addressed

Email: mgen@ staff.kanazawa-u.ac.jp; chak.k.chan@cityu.edu.hk

Telephone: + (852)-3442-5593

Fax: +(852)-3442-068 


\section{List of the supporting information:}

Text S1: Surface propensity of halide ions.

Text S2: Irradiation intensity of LED.

Text S3: Quantifying sulfate production during reactions and initial nitrate concentration.

Text S4: Characteristic time of mass transport and aqueous-phase oxidation of dissolved $\mathrm{SO}_{2}$.

Text S5: Additional mechanisms for sulfate production in the presence of $\mathrm{NaHCO}_{3}$ or Oxlac in air.

Text S6: Calculation of reactive uptake coefficient of $\mathrm{SO}_{2}$ for sulfate production.

Table S1: Mechanisms of sulfate formation pathways in this study.

Table S2: Initial concentration of $\mathrm{X}^{-}\left(\mathrm{X}^{-}=\mathrm{Cl}^{-}, \mathrm{Br}^{-}\right.$, and $\left.\mathrm{I}^{-}\right)$and $\mathrm{NO}_{3}^{-}$at $\left[\mathrm{X}^{-}\right]_{0} /\left[\mathrm{NO}_{3}{ }^{-}\right]_{0}$ of 0.2 in $\mathrm{N}_{2}$.

Table S3: Reactive uptake coefficients of $\mathrm{SO}_{2}$ for sulfate production in the presence of halide ions and $\mathrm{OH}$ scavengers.

Table S4: Equilibrium constants or mass transport coefficients.

Figure S1: Type of solvent cage in bulk phase and at the interface.

Figure S2: Schematic of experimental setup.

Figure S3: Raman spectrum of particulate 2NB dissolved into methanol.

Figure S4: Absorbance of $2 \mathrm{NB}$ as a function of [2NB] and cuvette path length, and photodecay plot of 2NB.

Figure S5: Calibration plot of ammonium sulfate and ammonium nitrate.

Figure S6: Particle pH of pure $\mathrm{NH}_{4} \mathrm{NO}_{3}, \mathrm{NH}_{4} \mathrm{NO}_{3}+\mathrm{NH}_{4} \mathrm{Cl}, \mathrm{NH}_{4} \mathrm{NO}_{3}+\mathrm{NH}_{4} \mathrm{Br}$, and $\mathrm{NH}_{4} \mathrm{NO}_{3}+$ $\mathrm{NH}_{4} \mathrm{I}$ as a function of uptake time in $\mathrm{N}_{2}$.

Figure S7: Mass transport of $\mathrm{SO}_{2}$ in gas phase and of dissolved $\mathrm{SO}_{2}$ in aqueous phase, respectively. Figure S8: Sulfate production rates of pure $\mathrm{NH}_{4} \mathrm{NO}_{3}$ and mixture of $\mathrm{NH}_{4} \mathrm{NO}_{3}+\mathrm{NaHCO}_{3}, \mathrm{NH}_{4} \mathrm{NO}_{3}$ + Oxlac, and $\mathrm{NH}_{4} \mathrm{NO}_{3}+\mathrm{NH}_{4} \mathrm{Cl}$ in air. 
Figure S9: Raman spectra of $\mathrm{NaHCO}_{3}$ in the presence of $\mathrm{SO}_{2}$ under dark in air and $\mathrm{N}_{2}$.

Figure S10: Sulfate production rates at initial molar ratio of $\mathrm{OH}$ scavenger to $\mathrm{NO}_{3}{ }^{-}$of $0,0.2$ and 1 in $\mathrm{N}_{2}$ condition.

Figure S11: Initial concentration of $\mathrm{NO}_{3}{ }^{-}, \mathrm{Cl}^{-}, \mathrm{NH}_{4}{ }^{+}, \mathrm{H}^{+}$and total ions at different $\left[\mathrm{Cl}^{-}\right]_{0} /\left[\mathrm{NO}_{3}{ }^{-}\right]_{0}$ based on E-AIM model.

Figure S12: Sensitive test of contribution of $\mathrm{N}(\mathrm{III}), \mathrm{NO}_{2}$, and $\mathrm{OH}$ pathway to sulfate production under different $\mathrm{pH}$ in kinetic model.

Figure S13: Model results of $\mathrm{N}(\mathrm{III}), \mathrm{NO}_{2}$, and $\mathrm{OH}$ radical concentration as a function of uptake time in different chemical systems.

Figure S14: Nitrate photolysis rate constant as a function of $\left[\mathrm{Cl}^{-}\right]_{0} /\left[\mathrm{NO}_{3}{ }^{-}\right] 0$ under $\mathrm{N}_{2}$ condition. 
Text S1. Surface propensity of halide ions.

Surface propensity refers to the tendency that ions prefer to reside near the air-liquid interface than in the bulk phase, with high surface propensity corresponding to surface ion concentration higher (by several times in some cases) than that of the bulk phase. Large and polarizable anions (e.g. $\mathrm{Cl}^{-}, \mathrm{Br}^{-}$, and $\mathrm{I}^{-}$) can be present and even enrich at the surface based on classical molecular dynamic (MD) model. ${ }^{1,2}$ It has been reported that all halide ions have high surface propensities, which is in accord with the polarizability of halide ions, and $\mathrm{I}^{-}$has a higher surface propensity than those of $\mathrm{Br}^{-}$and $\mathrm{Cl}^{-}{ }^{3}$

Text S2. Calculation of irradiation intensity of LED at the wavelength of $300 \mathrm{~nm}$.

To examine the irradiation intensity of LED, 2-nitrobenzaldehyde (2NB) as a photochemically sensitive and thermally robust actinometer was used in this study. ${ }^{4}$ We dissolved $2 \mathrm{NB}$ into methanol instead of water due to the limitation of $2 \mathrm{NB}$ solubility in water. Analogous to the particulate nitrate photolysis experiment in flow cell, particulate $2 \mathrm{NB}$ was deposited onto the bottom window of flow cell. Raman spectrum of 2NB is shown in Figure S3, and the peak area of 2NB $\left(\sim 1345 \mathrm{~cm}^{-1}\right)$ is normalized by peak area of methanol $\left(\sim 1040 \mathrm{~cm}^{-1}\right)$. The photodecay rate constant of $2 \mathrm{NB}, j(2 \mathrm{NB})$, was determined as the negative of the slope of blue line in Figure S4 based on Equation 1.

$\ln \left(\frac{[A(2 \mathrm{NB}) / A(\mathrm{MeOH})]_{\mathrm{t}}}{[A(2 \mathrm{NB}) / A(\mathrm{MeOH})]_{0}}\right)=-j(2 \mathrm{NB}) \times \mathrm{t}$

(Equation 1)

where $[A(2 \mathrm{NB}) / A(\mathrm{MeOH})]_{0}$ and $[A(2 \mathrm{NB}) / A(\mathrm{MeOH})]_{\mathrm{t}}$ are Raman peak area ratio of $2 \mathrm{NB}$ to $\mathrm{MeOH}$ at initial time and at different photolysis time, respectively.

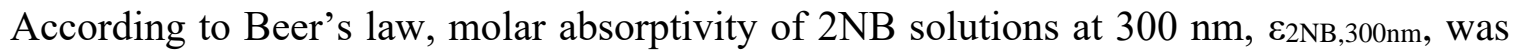
calculated as the slope of a linear regression of measured absorbance versus the $2 \mathrm{NB}$ concentration and cuvette path length (Figure S4) based on Equation 2:

$A b s .=\varepsilon_{2 \mathrm{NB}, 300 \mathrm{~nm}} \cdot l \cdot[2 \mathrm{NB}]$

(Equation 2) 
where $A b s$. is absorbance of $2 \mathrm{NB}$ at $300 \mathrm{~nm} ; l$ is cuvette path length $(=1 \mathrm{~cm})$; $[2 \mathrm{NB}]$ is $2 \mathrm{NB}$ concentration.

Hence, photon flux of LED can be calculated based on Equation 3:

$I_{300 \mathrm{~nm}}=\frac{j(2 \mathrm{NB})}{2.303 \times \varepsilon_{2 \mathrm{NB}, 300 \mathrm{~nm}} \times \phi_{2 \mathrm{NB}, 300 \mathrm{~nm}}} \cdot N_{\mathrm{A}}$

where $I_{300 \mathrm{~nm}}$ (photons $\mathrm{cm}^{-2} \mathrm{~s}^{-1}$ ) is irradiation intensity of LED at $300 \mathrm{~nm} . \varepsilon_{2 \mathrm{NB}, 300 \mathrm{~nm}}$ and $\phi_{2 \mathrm{NB}, 300 \mathrm{~nm}}$ are molar absorptivity and quantum yield of $2 \mathrm{NB}$, respectively. $\varepsilon 2 \mathrm{NB}, 300 \mathrm{~nm}$ was calculated based on absorbance of $2 \mathrm{NB}$ at $300 \mathrm{~nm}\left(\sim 1154.549 \mathrm{M}^{-1} \mathrm{~cm}^{-1}\right) . \phi_{2 \mathrm{NB}, \lambda}$ for liquid studies is estimated to be around 0.41 based on previous study. ${ }^{4} j(2 \mathrm{NB})$ is decay rate constant of $2 \mathrm{NB}$ in particle $(\sim 0.00296$ $\mathrm{s}^{-1}$ ) measured using Raman spectroscopy. $N_{\mathrm{A}}$ is the Avogadro constant.

Text S3. Method of quantifying sulfate concentration during reactions and initial nitrate concentration.

To quantify the sulfate concentration, the calibration curve of sulfate concentration as a function of the peak area ratio of $v\left(\mathrm{SO}_{4}{ }^{2-}\right)$ to $v(\mathrm{OH})_{\text {water, which was established based on Raman }}$ spectra of standard $\left(\mathrm{NH}_{4}\right)_{2} \mathrm{SO}_{4}$ solution at different concentrations (Figure $\mathrm{S} 5$ ). In this study, the upper limit of sulfate concentration quantified is $3 \mathrm{M}$ due to the limitation of linearity of calibration curve. Herein, sulfate concentration was determined based on Equation 4, where $k_{\mathrm{SO}_{4}^{2-}}$ is slope of 30.158 M in Figure S5.

$\left[\mathrm{SO}_{4}^{2-}\right]=k_{\mathrm{SO}_{4}^{2-}} \cdot \frac{A\left(\mathrm{SO}_{4}^{2-}\right)}{A(\mathrm{OH})}$

(Equation 4)

Analogous to the calculation of sulfate production, initial nitrate concentration in the presence of $\mathrm{Br}^{-}$or $\mathrm{I}^{-}$was calculated based on Equation 5. The calibration curve is displayed in Figure S5 with slope of 29.205 M.

$\left[\mathrm{NO}_{3}^{-}\right]=k_{\mathrm{NO}_{3}^{-}} \cdot \frac{A\left(\mathrm{NO}_{3}^{-}\right)}{A(\mathrm{OH})}$

(Equation 5) 
Text S4. Characteristic time of mass transport of gas phase and aqueous phase, and of aqueous oxidation of dissolved $\mathrm{SO}_{2}$.

Heterogeneous oxidation of dissolved $\mathrm{SO}_{2}$ during nitrate photolysis at $300 \mathrm{~nm}$ will involve a series of following steps (Figure S7): ${ }^{5-7}$ (a) diffusion of $\mathrm{SO}_{2}$ from the gas phase to the air-particle interface; (b) dissolution of gas-phase $\mathrm{SO}_{2}$ molecules into the interface and mass transport across the interface; (c) hydrolysis and ionization of dissolved $\mathrm{SO}_{2}$ at the interface; (d) diffusion of dissolved $\mathrm{SO}_{2}$ from interface to aqueous phase; (e) oxidation of dissolved $\mathrm{SO}_{2}$ by oxidants produced from nitrate photolysis. Herein, we compared the rates of mass transport in the gas phase, at the air-particle interface, and in the aqueous phase to quantify the impact of mass transport on the rate of aqueous-phase reactions.

First, we investigated the characteristic time to achieve equilibrium of heterogeneous uptake of $\mathrm{SO}_{2}$ and dissociation of dissolved $\mathrm{SO}_{2}$ at the air-particle interface, which can be calculated based on Equations 6 and 7, respectively:

$$
\mathrm{t}_{\mathrm{p}}=\frac{2 \pi M_{\mathrm{SO} 2} \mathrm{RT} D_{\mathrm{aq}}\left(H_{\mathrm{SO} 2}^{*}\right)^{2}}{\alpha_{\mathrm{SO} 2}^{2}}
$$

where $\mathrm{MSO} 2, \mathrm{HSO}{ }^{*}$ and $\alpha_{\mathrm{SO} 2}$ are the molecular weight, Henry's law constant, and the accommodation coefficient of $\mathrm{SO}_{2}$, respectively; $D_{a q}$ is aqueous-phase diffusion coefficient of $\mathrm{HSO}_{3}{ }^{-} ; \mathrm{R}$ and $\mathrm{T}$ are the gas constant and experimental temperature (=298.15 K), respectively. In this study, the time to reach equilibrium of heterogeneous uptake of $\mathrm{SO}_{2}$ at the interface is estimated to be $1.60 \times 10^{-8} \mathrm{~s}$. Note that heterogenous uptake rate at the interface is sufficiently rapid, so that assumption of instantaneous interfacial equilibrium is valid. Furthermore, subsequent to heterogeneous uptake of $\mathrm{SO}_{2}$ is dissociation reaction of dissolved $\mathrm{SO}_{2}$ to produce $\mathrm{HSO}_{3}{ }^{-}$, which is assumed to be the main dissociation reaction at low $\mathrm{pH}$ in this study. ${ }^{7}$ Herein, the characteristic time of aqueous dissociation reaction can be expressed as follows: ${ }^{7}$

$\mathrm{t}_{\mathrm{i}}=\frac{1}{k_{\mathrm{f}}+k_{\mathrm{r}}\left[\mathrm{H}^{+}\right]_{\mathrm{e}}+k_{\mathrm{r}}\left[\mathrm{HSO}_{3}^{-}\right]_{\mathrm{e}}}$

where $k_{\mathrm{f}}$ and $k_{\mathrm{r}}$ are the reaction constant of the first-order forward reaction and reaction constant of the second-order reverse reaction, respectively; $\left[\mathrm{H}^{+}\right]_{\mathrm{e}}$ and $\left[\mathrm{HSO}_{3}{ }^{-}\right]_{\mathrm{e}}$ are the concentrations of $\mathrm{H}^{+}$ 
and $\mathrm{HSO}_{3}{ }^{-}$, respectively, after achieving equilibrium. Therefore, the time to achieve equilibrium of aqueous dissociation is around $1.03 \times 10^{-7} \mathrm{~s}$ as calculated based on Equation 7.

Because the characteristic time to achieve equilibrium at the interface and of aqueous dissociation reaction are short enough, we assume that aqueous ionic equilibria can be hold at all points in particles and these characteristic times no longer need to be considered. ${ }^{7}$ Thus, we now compare the rate of mass transport with the aqueous-phase reaction rate to evaluate whether there is limitation on aqueous-phase reactions. When accommodation coefficient $\geq 0.1$, gas-phase diffusion is more restrictive for atmospheric particles compared to interfacial mass transport regardless of particle size. ${ }^{7}$ Since accommodation coefficient of $\mathrm{SO}_{2}$ is around 0.11 , interfacial mass transport can be ignored compared to gas-phase diffusion and aqueous-phase diffusion. Herein, we calculate the characteristic time of gas-phase diffusion and aqueous-phase diffusion based on Equations 8 and 9, respectively:

$\mathrm{t}_{\mathrm{g}}=\frac{\mathrm{R}_{\mathrm{p}}^{2}}{4 D_{\mathrm{g}}}$

(Equation 8)

$\mathrm{t}_{\mathrm{aq}}=\frac{\mathrm{R}_{\mathrm{p}}^{2}}{\pi^{2} D_{\mathrm{aq}}}$

(Equation 9)

where $t_{\mathrm{g}}$ and $\mathrm{t}_{\mathrm{aq}}$ are characteristic time of gas-phase diffusion and aqueous-phase diffusion, respectively; $\mathrm{R}_{p}$ is radius of particle in this study; $D_{g}$ and $D_{a q}$ are gas-phase diffusivity of $\mathrm{SO}_{2}$ and aqueous-phase diffusion coefficient of $\mathrm{HSO}_{3}{ }^{-}$, respectively. Hence, the characteristic time of gas phase diffusion and aqueous phase diffusion are $1.55 \times 10^{-5} \mathrm{~s}$ and $5.18 \times 10^{-2} \mathrm{~s}$, respectively.

To further evaluate whether mass transport constrains the aqueous-phase reactions, we calculated the characteristic time for aqueous-phase chemical reactions based on the Equation 10:

$\mathrm{t}_{\mathrm{r}}=-\frac{\left[\mathrm{HSO}_{3}^{-}\right]}{\mathrm{d}\left[\mathrm{HSO}_{3}^{-}\right] / \mathrm{dt}}$

(Equation 10)

where $\left[\mathrm{HSO}_{3}{ }^{-}\right]$is the concentration of $\mathrm{HSO}_{3}{ }^{-} ; \mathrm{d}\left[\mathrm{HSO}_{3}{ }^{-}\right] / \mathrm{dt}$ is oxidation rate of $\mathrm{HSO}_{3}{ }^{-}$. Here, we assumed that $\mathrm{HSO}_{3}{ }^{-}$can be oxidized into sulfate production completely and radical chain reactions are fast. We used sulfate production rate in the presence of $\mathrm{NaHCO}_{3}\left(\sim 5.28 \times 10^{-5} \mathrm{M} \mathrm{s}^{-1}\right)$ and I- $(\sim$ $1.24 \times 10^{-4} \mathrm{M} \mathrm{s}^{-1}$ ) in $\mathrm{N}_{2}$ to represent minimum and maximum oxidation rates of $\mathrm{HSO}_{3}{ }^{-}$in this study, 
respectively. Therefore, the characteristic time for aqueous-phase reactions varies from $4.98 \times 10^{-3}$ s to $7.37 \times 10^{-2} \mathrm{~s}$.

In conclusion, interface diffusion and gas-phase diffusion rate are sufficiently fast, which cannot constrain the oxidation reaction. Note that aqueous-phase diffusion rate and aqueous-phase reaction rate give comparable characteristic time. However, such characteristic time are still short enough compared to actual experiment time $(\sim 24 \mathrm{~h})$, hence, mass transport cannot restrict oxidation process of dissolved $\mathrm{SO}_{2}$. The equilibrium constants and mass transport coefficients are listed in Table S4.

Text S5. Additional mechanisms in sulfate production in the presence of $\mathrm{NaHCO}_{3}$ or oxlac in air.

Figure $\mathrm{S} 8$ shows that $\mathrm{NH}_{4} \mathrm{NO}_{3}+\mathrm{NH}_{4} \mathrm{Cl}$ particles exhibit the highest sulfate production rate $\left(84 \pm 1.3 \mu \mathrm{M} \mathrm{s}^{-1}\right)$, whereas the presence of $\mathrm{NaHCO}_{3}\left(56 \pm 2.6 \mu \mathrm{M} \mathrm{s}^{-1}\right)$ and oxlac $\left(62 \pm 5.7 \mu \mathrm{M} \mathrm{s}^{-1}\right)$ also show only slightly higher sulfate production than pure $\mathrm{NH}_{4} \mathrm{NO}_{3}\left(51 \pm 0.9 \mu \mathrm{M} \mathrm{s}^{-1}\right)$ in air. This result suggests that the accumulation of $\mathrm{N}(\mathrm{III})$ due to $\mathrm{OH}$ scavenging may play a role in the increased sulfate production rate. Alternatively, other mechanisms may also contribute to the increased sulfate production in the presence of $\mathrm{NaHCO}_{3}$ or oxlac in air. They include direct oxidation of $\mathrm{SO}_{2}$ by $\mathrm{HCO}_{3}{ }^{-}$(pathway 5 in Figure 1 in the main text) ${ }^{8}$ and $\mathrm{SO}_{2}$ oxidation by $\mathrm{H}_{2} \mathrm{O}_{2}$ produced from superoxide $\left(\mathrm{O}_{2}{ }^{-}\right),{ }^{9,10}$ a product of the reaction of oxlac with $\mathrm{OH}$ in the presence of $\mathrm{O}_{2}$ (pathway 6 in Figure 1). ${ }^{9}$ Uptake experiments were performed with $\mathrm{NaHCO}_{3}$ particles under dark conditions while the other conditions were kept as the same as before in air and $\mathrm{N}_{2}$. Significant sulfate production was found in the presence of $\mathrm{O}_{2}$, but no sulfate production was observed in $\mathrm{N}_{2}$ condition (Figure S9), which indicates that the presence of $\mathrm{HCO}_{3}{ }^{-}$can promote the formation of sulfate production in the presence of $\mathrm{O}_{2}$.

Text S6. Calculation of reactive uptake coefficient of $\mathrm{SO}_{2}$ for sulfate production.

We calculated reactive uptake coefficient of $\mathrm{SO}_{2}, \gamma_{\mathrm{SO} 2}$, for sulfate production using sulfate production rate, $\mathrm{d}\left[\mathrm{SO}_{4}{ }^{2-}\right] / \mathrm{dt}$, obtained from the linear fitting to the plot of $\left[\mathrm{SO}_{4}{ }^{2-}\right]$ versus uptake time (Equation 11). 
$\gamma_{\mathrm{SO} 2}=\frac{\mathrm{d}\left[\mathrm{SO}_{4}^{2-}\right]}{\mathrm{dt}} / \mathrm{Z}$

(Equation 11)

$\mathrm{Z}=\frac{1}{4} \omega_{\mathrm{SO}_{2}} A_{\mathrm{s}}\left[\mathrm{SO}_{2}\right]$

$\omega_{\mathrm{SO}_{2}}=\sqrt{8 \mathrm{RT} / \pi M_{\mathrm{SO}_{2}}}$

$\mathrm{A}_{\mathrm{s}}=4 \pi r_{\mathrm{p}}^{2} \times\left(4 \pi r_{\mathrm{p}}^{3} / 3\right)^{-1}$

Where $\omega_{\mathrm{SO}_{2}}$ and $\mathrm{MSO}_{2}$ are the thermal velocity and the molecular weight of $\mathrm{SO}_{2}$, respectively. $\left[\mathrm{SO}_{2}\right]$ is the gas phase concentration. $A_{s}$ and $r_{p}$ are the surface aera of a particle per the volume, and the particle radius, respectively. The values of reactive uptake coefficient of $\mathrm{SO}_{2}$ are listed in the Table S3. 
Table S1. Mechanisms for sulfate formation pathways in this study.

\begin{tabular}{|c|c|c|c|}
\hline \multicolumn{2}{|r|}{ Reaction } & $\begin{array}{l}\text { Rate constant } \\
\left(\mathrm{M}^{-1} \mathrm{~s}^{-1} \text { or s}^{-1}\right)\end{array}$ & $\begin{array}{l}\text { Note / } \\
\text { reference }\end{array}$ \\
\hline $1^{*}$ & $\begin{aligned} \mathrm{NO}_{3}^{-}+h v & \rightarrow \mathrm{NO}_{2}+\mathrm{OH} \\
& \rightarrow \mathrm{NO}_{2}^{-}+\mathrm{O}\left({ }^{3} \mathrm{P}\right)\end{aligned}$ & $j_{\mathrm{NO} O}-$ & This study \\
\hline $2 *$ & $\begin{array}{c}\mathrm{N}(\mathrm{III})+h v+\mathrm{H}^{+} \rightarrow \mathrm{NO}+\mathrm{OH} \\
\mathrm{N}(\mathrm{III}): \mathrm{NO}_{2}-/ \mathrm{HNO}_{2}\end{array}$ & $j_{\mathrm{N}(\mathrm{III})}$ & This study \\
\hline \multicolumn{4}{|c|}{ OH mechanisms } \\
\hline $3 *$ & $\mathrm{OH}+\mathrm{HSO}_{3}{ }^{-} \rightarrow \mathrm{SO}_{3}{ }^{-}+\mathrm{H}_{2} \mathrm{O}$ & $4.5 \times 10^{9}$ & 7 \\
\hline 4 & $\mathrm{SO}_{3}^{-}+\mathrm{O}_{2} \rightarrow \mathrm{SO}_{5}^{-}$ & $1.5 \times 10^{9}$ & 7 \\
\hline 5 & $\mathrm{SO}_{5}^{-}+\mathrm{HSO}_{3}^{-} \rightarrow \mathrm{HSO}_{5}^{-}+\mathrm{SO}_{3}^{-}$ & $2.5 \times 10^{4}$ & 7 \\
\hline 6 & $\mathrm{SO}_{5}{ }^{-}+\mathrm{HSO}_{3}^{-} \rightarrow \mathrm{SO}_{4}^{-}+\mathrm{SO}_{4}{ }^{2-}+\mathrm{H}^{+}$ & $7.5 \times 10^{4}$ & 7 \\
\hline $7 *$ & $\begin{array}{c}\mathrm{SO}_{4}^{-}+\mathrm{HSO}_{3}^{-} \rightarrow \mathrm{SO}_{4}{ }^{2-}+\mathrm{SO}_{3}^{-}+\mathrm{H}^{+} \\
\mathrm{k}_{7}\left[\mathrm{SO}_{4}^{-}\right]\left[\mathrm{HSO}_{3}^{-}\right]^{\mathrm{c}}\end{array}$ & $\mathrm{k}_{7}=7.5 \times 10^{8}$ & 7 \\
\hline 8 & $\mathrm{SO}_{5}{ }^{-}+\mathrm{SO}_{5}{ }^{-} \rightarrow 2 \mathrm{SO}_{4}{ }^{2-}+\mathrm{O}_{2}$ & $6.0 \times 10^{8}$ & 7 \\
\hline 9 & $\mathrm{SO}_{5}{ }^{-}+\mathrm{SO}_{5}^{-} \rightarrow \mathrm{S}_{2} \mathrm{O}_{8}{ }^{2-}+\mathrm{O}_{2}$ & $1.4 \times 10^{8}$ & 7 \\
\hline $10^{*}$ & $\mathrm{SO}_{3}{ }^{-}+\mathrm{SO}_{3}{ }^{-} \rightarrow \mathrm{S}_{2} \mathrm{O}_{6}{ }^{2-}$ & $7.0 \times 10^{8}$ & 7 \\
\hline $11^{*}$ & $\mathrm{SO}_{4}^{-}+\mathrm{SO}_{4}^{-} \rightarrow \mathrm{S}_{2} \mathrm{O}_{8}^{2-}$ & $4.5 \times 10^{8}$ & 7 \\
\hline $12^{*}$ & $\begin{array}{c}\mathrm{SO}_{4}^{-}+\mathrm{NO}_{3}^{-} \rightarrow \mathrm{SO}_{4}^{2-}+\mathrm{NO}_{3} \\
\mathrm{k}_{12}\left[\mathrm{SO}_{4}^{-}\right]\left[\mathrm{NO}_{3}^{-}\right]^{\mathrm{c}}\end{array}$ & $\mathrm{k}_{12}=5.0 \times 10^{4}$ & 11 \\
\hline 13 & $\mathrm{HSO}_{5}^{-}+\mathrm{OH} \rightarrow \mathrm{SO}_{5}^{-}+\mathrm{H}_{2} \mathrm{O}$ & $1.7 \times 10^{7}$ & 7 \\
\hline 14 & $\mathrm{HSO}_{5}{ }^{-}+\mathrm{SO}_{4}^{-} \rightarrow \mathrm{SO}_{5}^{-}+\mathrm{SO}_{4}{ }^{2-}+\mathrm{H}^{+}$ & $<1.0 \times 10^{5}$ & 7 \\
\hline $15^{\mathrm{a}}$ & $\mathrm{HSO}_{5}{ }^{-}+\mathrm{HSO}_{3}{ }^{-}+\mathrm{H}^{+} \rightarrow 2 \mathrm{SO}_{4}{ }^{2-}+3 \mathrm{H}^{+}$ & $7.1 \times 10^{6}$ & 7 \\
\hline $16^{*}$ & $\mathrm{OH}+\mathrm{OH} \rightarrow \mathrm{H}_{2} \mathrm{O}_{2}$ & $5.5 \times 10^{9}$ & 12 \\
\hline \multicolumn{4}{|c|}{$\mathrm{NO}_{2}$ mechanisms } \\
\hline $17 *$ & $\mathrm{NO}_{2}+\mathrm{OH} \rightarrow \mathrm{NO}_{3}^{-}+\mathrm{H}^{+}$ & $4.5 \times 10^{9}$ & 13 \\
\hline $18^{*}$ & $\mathrm{NO}_{2}+\mathrm{NO}+\mathrm{H}_{2} \mathrm{O} \rightarrow 2 \mathrm{NO}_{2}^{-}+2 \mathrm{H}^{+}$ & $2.0 \times 10^{8}$ & 7 \\
\hline 19 & $\mathrm{NO}_{2}+\mathrm{O}_{3} \rightarrow \mathrm{NO}_{3}+\mathrm{O}_{2}$ & $1.9 \times 10^{4}$ & 14 \\
\hline $20 *$ & $\mathrm{NO}_{3}+\mathrm{NO}_{2} \rightarrow \mathrm{N}_{2} \mathrm{O}_{5}$ & $1.0 \times 10^{9}$ & 15 \\
\hline
\end{tabular}




\begin{tabular}{|c|c|c|c|}
\hline $21^{*}$ & $\mathrm{HSO}_{3}{ }^{-}+\mathrm{NO}_{3} \rightarrow \mathrm{NO}_{3}^{-}+\mathrm{H}^{+}+2 \mathrm{SO}_{3}^{-}$ & $1.4 \times 10^{9}$ & 11 \\
\hline $22 *$ & $\mathrm{NO}_{2}+\mathrm{NO}_{2}+\mathrm{H}_{2} \mathrm{O} \rightarrow \mathrm{NO}_{2}^{-}+\mathrm{NO}_{3}^{-}+2 \mathrm{H}^{+}$ & $1.0 \times 10^{8}$ & 7 \\
\hline $23^{*}$ & $\begin{array}{c}2 \mathrm{NO}_{2}+\mathrm{HSO}_{3}^{-}+\mathrm{H}_{2} \mathrm{O} \rightarrow 3 \mathrm{H}^{+}+2 \mathrm{NO}_{2}^{-}+\mathrm{SO}_{4}^{2-} \\
\mathrm{k}_{23}\left[\mathrm{HSO}_{3}^{-}\right]\left[\mathrm{NO}_{2}\right]^{\mathrm{c}}\end{array}$ & $\mathrm{k}_{23}=2.0 \times 10^{6}$ & 7 \\
\hline \multicolumn{4}{|c|}{$\mathrm{NO}_{2}{ }^{-}$mechanisms } \\
\hline $24 *$ & $\mathrm{NO}+\mathrm{OH} \rightarrow \mathrm{NO}_{2}^{-}+\mathrm{H}^{+}$ & $2.0 \times 10^{10}$ & 7 \\
\hline $25^{*}$ & $\mathrm{HNO}_{2}+\mathrm{OH} \rightarrow \mathrm{NO}_{2}+\mathrm{H}_{2} \mathrm{O}$ & $1.0 \times 10^{9}$ & 7 \\
\hline 26 & $\mathrm{HNO}_{2}+\mathrm{H}_{2} \mathrm{O}_{2}+\mathrm{H}^{+} \rightarrow \mathrm{NO}_{3}{ }^{-}+2 \mathrm{H}^{+}+\mathrm{H}_{2} \mathrm{O}$ & $6.3 \times 10^{3} \times\left[\mathrm{H}^{+}\right]$ & 7 \\
\hline 27 & $\mathrm{HSO}_{5}^{-}+\mathrm{NO}_{2}^{-} \rightarrow \mathrm{HSO}_{4}^{-}+\mathrm{NO}_{3}^{-}$ & 0.31 & 7 \\
\hline $28^{*}$ & $\begin{array}{c}\mathrm{SO}_{4}^{-}+\mathrm{NO}_{2}^{-} \rightarrow \mathrm{SO}_{4}^{2-}+\mathrm{NO}_{2} \\
\mathrm{k}_{28}\left[\mathrm{SO}_{4}^{-}\right]\left[\mathrm{NO}_{2}^{-}\right]^{\mathrm{c}}\end{array}$ & $\mathrm{k}_{28}=8.8 \times 10^{8}$ & 7 \\
\hline 29 & $\mathrm{NO}_{2}^{-}+\mathrm{O}_{3} \rightarrow \mathrm{NO}_{3}^{-}+\mathrm{O}_{2}$ & $5.0 \times 10^{5}$ & 7 \\
\hline $30 *$ & $\mathrm{NO}_{2}{ }^{-}+\mathrm{NO}_{3} \rightarrow \mathrm{NO}_{3}{ }^{-}+\mathrm{NO}_{2}$ & $1.2 \times 10^{9}$ & 16 \\
\hline $31^{*}$ & $\begin{array}{c}\mathrm{N}(\mathrm{III})+\mathrm{HSO}_{3}^{-}+\mathrm{H}^{+} \rightarrow \mathrm{NOH}+\mathrm{SO}_{4}{ }^{2-}+2 \mathrm{H}^{+} \\
\mathrm{k}_{31}\left[\mathrm{HSO}_{3}^{-}\right][\mathrm{N}(\mathrm{III})]^{\mathrm{c}}\end{array}$ & $\begin{array}{c}\mathrm{k}_{31}=3.8 \times 10^{3} \times \\
{\left[\mathrm{H}^{+}\right]}\end{array}$ & 17 \\
\hline \multicolumn{4}{|c|}{$\mathrm{O}_{3}$ mechanisms } \\
\hline 32 & $\mathrm{O}\left({ }^{3} \mathrm{P}\right)+\mathrm{O}_{2} \rightarrow \mathrm{O}_{3}$ & $4.0 \times 10^{9}$ & 12 \\
\hline 33 & $\mathrm{O}_{3}+\mathrm{OH} \rightarrow \mathrm{HO}_{2}+\mathrm{O}_{2}$ & $1.1 \times 10^{8}$ & 12 \\
\hline 34 & $\mathrm{OH}+\mathrm{OH} \rightarrow \mathrm{H}_{2} \mathrm{O}_{2}$ & $6.0 \times 10^{9}$ & 12 \\
\hline 35 & $\mathrm{OH}+\mathrm{H}_{2} \mathrm{O}_{2} \rightarrow \mathrm{H}_{2} \mathrm{O}+\mathrm{HO}_{2}$ & $2.7 \times 10^{7}$ & 7 \\
\hline 36 & $\mathrm{OH}+\mathrm{HO}_{2} \rightarrow \mathrm{H}_{2} \mathrm{O}+\mathrm{O}_{2}$ & $7.0 \times 10^{9}$ & 7 \\
\hline 37 & $\mathrm{HO}_{2}+\mathrm{HO}_{2} \rightarrow \mathrm{H}_{2} \mathrm{O}_{2}+\mathrm{O}_{2}$ & $8.6 \times 10^{5}$ & 7 \\
\hline 38 & $\mathrm{O}_{3}+\mathrm{HO}_{2} \rightarrow \mathrm{H}^{+}+\mathrm{O}_{2}+\mathrm{O}_{3}^{-}$ & $1.6 \times 10^{9}$ & 12 \\
\hline 39 & $\mathrm{NO}_{3}+\mathrm{HO}_{2} \rightarrow \mathrm{NO}_{3}{ }^{-}+\mathrm{O}_{2}$ & $4.5 \times 10^{9}$ & 7 \\
\hline 40 & $\mathrm{HO}_{2}+\mathrm{H}_{2} \mathrm{O}_{2} \rightarrow \mathrm{OH}+\mathrm{O}_{2}+\mathrm{H}_{2} \mathrm{O}$ & 0.5 & 7 \\
\hline 41 & $\mathrm{O}_{3}+\mathrm{H}_{2} \mathrm{O}_{2} \rightarrow \mathrm{OH}+\mathrm{HO}_{2}+2 \mathrm{O}_{2}$ & 0.025 & 18 \\
\hline 42 & $\mathrm{NO}_{3}+\mathrm{H}_{2} \mathrm{O}_{2} \rightarrow \mathrm{NO}_{3}^{-}+\mathrm{H}^{+}+\mathrm{HO}_{2}$ & $1.0 \times 10^{6}$ & 7 \\
\hline 43 & $\mathrm{SO}_{4}^{-}+\mathrm{HO}_{2} \rightarrow \mathrm{SO}_{4}^{2-}+\mathrm{H}^{+}+\mathrm{O}_{2}$ & $5.0 \times 10^{9}$ & 7 \\
\hline 44 & $\mathrm{SO}_{4}^{-}+\mathrm{H}_{2} \mathrm{O}_{2} \rightarrow \mathrm{SO}_{4}{ }^{2-}+\mathrm{H}^{+}+\mathrm{HO}_{2}$ & $1.2 \times 10^{7}$ & 7 \\
\hline 45 & $\mathrm{~S}(\mathrm{IV})+\mathrm{HO}_{2} \rightarrow \mathrm{S}(\mathrm{VI})+\mathrm{OH}$ & $1.0 \times 10^{6}$ & 7 \\
\hline
\end{tabular}




\begin{tabular}{|c|c|c|c|}
\hline $46^{*}$ & $\begin{array}{c}\mathrm{S}(\mathrm{IV})+\mathrm{H}_{2} \mathrm{O}_{2} \rightarrow \mathrm{S}(\mathrm{VI})+\mathrm{H}_{2} \mathrm{O} \\
\mathrm{k}_{46}[\mathrm{~S}(\mathrm{IV})]\left[\mathrm{H}_{2} \mathrm{O}_{2}\right]\left[\mathrm{H}^{+}\right] /\left(1+13\left[\mathrm{H}^{+}\right]\right)^{\mathrm{c}}\end{array}$ & $\mathrm{k}_{46}=7.45 \times 10^{7}$ & 7 \\
\hline 47 & $\begin{array}{c}\mathrm{S}(\mathrm{IV})+\mathrm{O}_{3} \rightarrow \mathrm{S}(\mathrm{VI})+\mathrm{O}_{2} \\
\left(\mathrm{k}_{47 \mathrm{a}}\left[\mathrm{SO}_{2} \cdot \mathrm{H}_{2} \mathrm{O}\right]+\mathrm{k}_{476}\left[\mathrm{HSO}_{3}{ }^{-}\right]+\mathrm{k}_{47 c}\left[\mathrm{SO}_{3}{ }^{2-}\right]\right)\left[\mathrm{O}_{3}\right]^{\mathrm{c}}\end{array}$ & $\begin{array}{l}\mathrm{k}_{47 \mathrm{a}}=2.4 \times 10^{4} \\
\mathrm{k} 47 \mathrm{~b}=3.7 \times 10^{5} \\
\mathrm{k} 47 \mathrm{c}=1.5 \times 10^{9}\end{array}$ & 7 \\
\hline \multicolumn{4}{|c|}{$\mathrm{Cl}^{-}$mechanisms } \\
\hline $48^{*}$ & $\mathrm{Cl}^{-}+\mathrm{OH} \rightarrow \mathrm{ClOH}^{-}$ & $4.3 \times 10^{9}$ & 7 \\
\hline $49 * \mathrm{~b}$ & $\mathrm{ClOH}^{-} \rightarrow \mathrm{Cl}^{-}+\mathrm{OH}$ & $6.1 \times 10^{9}$ & 7 \\
\hline $50^{*}$ & $\mathrm{Cl}^{-}+\mathrm{ClOH}^{-} \rightarrow \mathrm{OH}^{-}+\mathrm{Cl}_{2}^{-}$ & $1.0 \times 10^{4}$ & 12 \\
\hline $51^{*}$ & $\mathrm{Cl}^{-}+\mathrm{HOCl}+\mathrm{H}^{+} \rightarrow \mathrm{Cl}_{2}+\mathrm{H}_{2} \mathrm{O}$ & $1.8 \times 10^{4}$ & 19 \\
\hline $52^{*}$ & $\mathrm{Cl}_{2}+\mathrm{H}_{2} \mathrm{O} \rightarrow \mathrm{Cl}^{-}+\mathrm{HOCl}+\mathrm{H}^{+}$ & 11 & 19 \\
\hline $53^{*}$ & $\mathrm{Cl}^{-}+\mathrm{Cl} \rightarrow \mathrm{Cl}_{2}^{-}$ & $6.5 \times 10^{9}$ & 20 \\
\hline $54 * \mathrm{~b}$ & $\mathrm{Cl}_{2}^{-} \rightarrow \mathrm{Cl}+\mathrm{Cl}^{-}$ & $6.0 \times 10^{4}$ & 12 \\
\hline $55^{*}$ & $\mathrm{Cl}^{-}+\mathrm{Cl}_{2} \rightarrow \mathrm{Cl}_{3}^{-}$ & $2.0 \times 10^{4}$ & 12 \\
\hline $56^{* \mathrm{~b}}$ & $\mathrm{Cl}_{3}^{-} \rightarrow \mathrm{Cl}_{2}+\mathrm{Cl}^{-}$ & $1.1 \times 10^{5}$ & 12 \\
\hline 57 & $\mathrm{Cl}^{-}+\mathrm{O}_{3} \rightarrow \mathrm{O}_{2}+\mathrm{OCl}^{-}$ & $1.6 \times 10^{-3}$ & 12 \\
\hline $58 *$ & $\mathrm{Cl}^{-}+\mathrm{NO}_{3} \rightarrow \mathrm{NO}_{3}{ }^{-}+\mathrm{Cl}$ & $3.5 \times 10^{8}$ & 15 \\
\hline $59^{*}$ & $\mathrm{NO}_{3}{ }^{-}+\mathrm{Cl} \rightarrow \mathrm{NO}_{3}+\mathrm{Cl}^{-}$ & $1.0 \times 10^{8}$ & 15 \\
\hline 60 & $\mathrm{Cl}^{-}+\mathrm{HSO}_{5}^{-} \rightarrow \mathrm{SO}_{4}^{2-}+$ product & $1.8 \times 10^{-3}$ & 7 \\
\hline $61^{*}$ & $\begin{array}{c}\mathrm{Cl}^{-}+\mathrm{SO}_{4}^{-} \rightarrow \mathrm{SO}_{4}^{2-}+\mathrm{Cl} \\
\mathrm{k}_{61}\left[\mathrm{SO}_{4}^{-}\right]\left[\mathrm{Cl}^{-}\right]^{\mathrm{c}}\end{array}$ & $\mathrm{k}_{61}=2.0 \times 10^{8}$ & 7 \\
\hline $62^{*}$ & $\mathrm{SO}_{4}{ }^{2-}+\mathrm{Cl} \rightarrow \mathrm{Cl}^{-}+\mathrm{SO}_{4}^{-}$ & $2.5 \times 10^{8}$ & 21 \\
\hline $63^{*}$ & $\mathrm{Cl}+\mathrm{H}_{2} \mathrm{O} \rightarrow \mathrm{ClOH}^{-}+\mathrm{H}^{+}$ & $1.3 \times 10^{3}$ & 7 \\
\hline $64 *$ & $\mathrm{ClOH}^{-}+\mathrm{H}^{+} \rightarrow \mathrm{Cl}+\mathrm{H}_{2} \mathrm{O}$ & $2.1 \times 10^{10} \times\left[\mathrm{H}^{+}\right]$ & 7 \\
\hline $65^{*}$ & $\mathrm{Cl}+\mathrm{OH}^{-} \rightarrow \mathrm{ClOH}^{-}$ & $1.8 \times 10^{10}$ & 22 \\
\hline $66^{* b}$ & $\mathrm{ClOH}^{-} \rightarrow \mathrm{Cl}+\mathrm{OH}^{-}$ & 23 & 22 \\
\hline $67^{*}$ & $\mathrm{Cl}+\mathrm{Cl} \rightarrow \mathrm{Cl}_{2}$ & $8.8 \times 10^{7}$ & 12 \\
\hline $68^{*}$ & $\mathrm{Cl}+\mathrm{Cl}_{2} \rightarrow \mathrm{Cl}_{3}$ & $5.3 \times 10^{8}$ & 12 \\
\hline $69^{*}$ & $\mathrm{Cl}+\mathrm{Cl}_{2}^{-} \rightarrow \mathrm{Cl}_{2}+\mathrm{Cl}^{-}$ & $2.1 \times 10^{9}$ & 23 \\
\hline $70^{*}$ & $\mathrm{Cl}+\mathrm{HOCl} \rightarrow \mathrm{Cl}^{-}+\mathrm{ClO}+\mathrm{H}^{+}$ & $3.0 \times 10^{9}$ & 12 \\
\hline
\end{tabular}




\begin{tabular}{|c|c|c|c|}
\hline $71^{*}$ & $\mathrm{Cl}+\mathrm{HSO}_{3}{ }^{-} \rightarrow \mathrm{Cl}^{-}+\mathrm{SO}_{3}{ }^{-}+\mathrm{H}^{+}$ & $3.4 \times 10^{9}$ & 24 \\
\hline $72 *$ & $\mathrm{Cl}_{2}^{-}+\mathrm{Cl}_{2}^{-} \rightarrow \mathrm{Cl}_{2}+2 \mathrm{Cl}^{-}$ & $9.0 \times 10^{8}$ & 12 \\
\hline $73^{*}$ & $\mathrm{Cl}_{2}^{-}+\mathrm{Cl}_{2}^{-} \rightarrow \mathrm{Cl}_{3}^{-}+\mathrm{Cl}^{-}$ & $4.07 \times 10^{9}$ & 12 \\
\hline $74^{*}$ & $\mathrm{Cl}_{2}^{-}+\mathrm{OH} \rightarrow \mathrm{HOCl}+\mathrm{Cl}^{-}$ & $1.0 \times 10^{9}$ & 12 \\
\hline $75^{*}$ & $\mathrm{Cl}_{2}^{-}+\mathrm{NO}_{3} \rightarrow \mathrm{NO}_{3}^{-}+\mathrm{Cl}_{2}$ & $1.0 \times 10^{9}$ & 15 \\
\hline $76^{*}$ & $\mathrm{Cl}_{2}^{-}+\mathrm{NO}_{2}^{-} \rightarrow \mathrm{NO}_{2}+2 \mathrm{Cl}^{-}$ & $2.5 \times 10^{8}$ & 7 \\
\hline $77^{*}$ & $\mathrm{Cl}_{2}^{-}+\mathrm{HSO}_{3}^{-} \rightarrow \mathrm{SO}_{3}^{-}+2 \mathrm{Cl}^{-}+\mathrm{H}^{+}$ & $3.4 \times 10^{8}$ & 24 \\
\hline $78^{*}$ & $\mathrm{Cl}_{2}+\mathrm{OH} \rightarrow \mathrm{HOCl}+\mathrm{Cl}^{-}$ & $1.0 \times 10^{9}$ & 25 \\
\hline $79 * \mathrm{~b}$ & $\mathrm{HOCl} \rightarrow \mathrm{H}^{+}+\mathrm{OCl}^{-}$ & $1.41 \times 10^{3}$ & 12 \\
\hline $80^{*}$ & $\mathrm{H}^{+}+\mathrm{OCl}^{-} \rightarrow \mathrm{HOCl}$ & $5.0 \times 10^{10}$ & 12 \\
\hline $81^{*}$ & $\mathrm{HOCl}+\mathrm{OH} \rightarrow \mathrm{ClO}+\mathrm{H}_{2} \mathrm{O}$ & $1.21 \times 10^{9}$ & 12 \\
\hline $82^{*}$ & $\begin{array}{c}\mathrm{HOCl}+\mathrm{HSO}_{3}^{-} \rightarrow \mathrm{SO}_{4}{ }^{2-}+\mathrm{Cl}^{-}+2 \mathrm{H}^{+} \\
\mathrm{k}_{82}[\mathrm{HOCl}]\left[\mathrm{HSO}_{3}^{-}\right]^{\mathrm{c}}\end{array}$ & $\mathrm{k}_{82}=7.6 \times 10^{8}$ & 26 \\
\hline \multicolumn{4}{|c|}{ Br- mechanisms } \\
\hline $83^{*}$ & $\mathrm{Br}^{-}+\mathrm{OH} \rightarrow \mathrm{BrOH}^{-}$ & $1.0 \times 10^{10}$ & 27 \\
\hline $84 * \mathrm{~b}$ & $\mathrm{BrOH}^{-} \rightarrow \mathrm{Br}^{-}+\mathrm{OH}$ & $3.3 \times 10^{7}$ & 27 \\
\hline $85^{*}$ & $\mathrm{Br}^{-}+\mathrm{Br} \rightarrow \mathrm{Br}_{2}^{-}$ & $1.0 \times 10^{10}$ & 27 \\
\hline $86^{* \mathrm{~b}}$ & $\mathrm{Br}_{2}^{-} \rightarrow \mathrm{Br}^{-}+\mathrm{Br}$ & $1.9 \times 10^{4}$ & 28 \\
\hline $87^{*}$ & $\mathrm{Br}^{-}+\mathrm{Br}_{2} \rightarrow \mathrm{Br}_{3}^{-}$ & $9.6 \times 10^{8}$ & 29 \\
\hline $88 * \mathrm{~b}$ & $\mathrm{Br}_{3}^{-} \rightarrow \mathrm{Br}_{2}+\mathrm{Br}^{-}$ & $5.5 \times 10^{7}$ & 29 \\
\hline $89^{*}$ & $\mathrm{Br}^{-}+\mathrm{BrOH}^{-} \rightarrow \mathrm{OH}^{-}+\mathrm{Br}_{2}^{-}$ & $1.9 \times 10^{8}$ & 27 \\
\hline $90 * \mathrm{a}$ & $\mathrm{Br}^{-}+\mathrm{HOBr}+\mathrm{H}^{+} \rightarrow \mathrm{Br}_{2}+\mathrm{H}_{2} \mathrm{O}$ & $1.6 \times 10^{10}$ & 19 \\
\hline $91^{*}$ & $\mathrm{Br}_{2}+\mathrm{H}_{2} \mathrm{O} \rightarrow \mathrm{HOBr}+\mathrm{H}^{+}+\mathrm{Br}^{-}$ & $1.1 \times 10^{2}$ & 19 \\
\hline 92 & $\mathrm{Br}^{-}+\mathrm{SO}_{4}^{--} \rightarrow \mathrm{SO}_{4}^{2-}+\mathrm{Br}$ & $3.5 \times 10^{9}$ & 30 \\
\hline $93^{*}$ & $\mathrm{Br}+\mathrm{OH}^{-} \rightarrow \mathrm{BrOH}^{-}$ & $1.3 \times 10^{10}$ & 22 \\
\hline $94 * \mathrm{~b}$ & $\mathrm{BrOH}^{-} \rightarrow \mathrm{Br}+\mathrm{OH}^{-}$ & $4.2 \times 10^{6}$ & 22 \\
\hline $95^{*}$ & $\mathrm{Br}+\mathrm{H}_{2} \mathrm{O} \rightarrow \mathrm{BrOH}^{-}+\mathrm{H}^{+}$ & 1.36 & 22 \\
\hline $96^{*}$ & $\mathrm{BrOH}^{-}+\mathrm{H}^{+} \rightarrow \mathrm{H}_{2} \mathrm{O}+\mathrm{Br}$ & $4.4 \times 10^{10}$ & 22 \\
\hline $97^{*}$ & $\mathrm{Br}+\mathrm{Br} \rightarrow \mathrm{Br}_{2}$ & $1.0 \times 10^{9}$ & 30 \\
\hline $98^{*}$ & $\mathrm{Br}_{2}^{-}+\mathrm{Br}_{2}^{-} \rightarrow \mathrm{Br}_{3}^{-}+\mathrm{Br}^{-}$ & $2.0 \times 10^{9}$ & 31 \\
\hline
\end{tabular}




\begin{tabular}{|c|c|c|c|}
\hline $99 *$ & $\mathrm{Br}_{2}^{-}+\mathrm{Br} \rightarrow \mathrm{Br}_{2}+\mathrm{Br}^{-}$ & $2.0 \times 10^{9}$ & 30 \\
\hline $100^{*}$ & $\mathrm{Br}_{2}^{-}+\mathrm{NO}_{2}^{-} \rightarrow 2 \mathrm{Br}^{-}+\mathrm{NO}_{2}$ & $2.0 \times 10^{7}$ & 32 \\
\hline $101^{*}$ & $\mathrm{Br}_{2}^{-}+\mathrm{NO} \rightarrow \mathrm{NO}^{-}+\mathrm{Br}_{2}$ & $4.8 \times 10^{9}$ & 33 \\
\hline $102 *$ & $\mathrm{Br}_{2}^{-}+\mathrm{HSO}_{3}^{-} \rightarrow 2 \mathrm{Br}^{-}+\mathrm{H}^{+}+\mathrm{SO}_{3}^{-}$ & $6.9 \times 10^{7}$ & 34 \\
\hline $103^{*}$ & $\begin{array}{c}\mathrm{HOBr}+\mathrm{HSO}_{3}^{-} \rightarrow \mathrm{Br}^{-}+\mathrm{SO}_{4}^{2-}+2 \mathrm{H}^{+} \\
\mathrm{k}_{103}[\mathrm{HOBr}]\left[\mathrm{HSO}_{3}^{-}\right]^{\mathrm{c}}\end{array}$ & $\mathrm{k}_{103}=3.2 \times 10^{9}$ & 35 \\
\hline $104 *$ & $\mathrm{HOBr}+\mathrm{OH} \rightarrow \mathrm{BrO}+\mathrm{H}_{2} \mathrm{O}$ & $2.0 \times 10^{9}$ & 31 \\
\hline \multicolumn{4}{|c|}{ I' mechanisms } \\
\hline $105^{*}$ & $\mathrm{I}^{-}+h v \rightarrow\left[\mathrm{I}+\mathrm{e}^{-}\right]_{\text {cage }}$ & $3.1 \times 10^{-8}$ & $\begin{array}{c}\text { This } \\
\text { study }\end{array}$ \\
\hline $106^{*}$ & {$\left[\mathrm{I}+\mathrm{e}^{-}\right]_{\text {cage }}+\mathrm{H}^{+} \rightarrow \mathrm{I}+\mathrm{H}$} & $1.0 \times 10^{10}$ & 36 \\
\hline $107^{*}$ & $\mathrm{I}^{-}+\mathrm{OH} \rightarrow \mathrm{HOI}^{-}$ & $1.1 \times 10^{10}$ & 37 \\
\hline $108 * \mathrm{~b}$ & $\mathrm{HOI}^{-} \rightarrow \mathrm{I}^{-}+\mathrm{OH}$ & $2.25 \times 10^{6}$ & 38 \\
\hline $109^{*}$ & $\mathrm{I}^{-}+\mathrm{I} \rightarrow \mathrm{I}_{2}^{-}$ & $8.8 \times 10^{9}$ & 37 \\
\hline $110^{* \mathrm{~b}}$ & $\mathrm{I}_{2}^{-} \rightarrow \mathrm{I}+\mathrm{I}^{-}$ & $6.9 \times 10^{4}$ & 37 \\
\hline $111^{*}$ & $\mathrm{I}^{-}+\mathrm{NO}_{2} \rightarrow \mathrm{I}+\mathrm{NO}_{2}^{-}$ & $2.77 \times 10^{6}$ & 39 \\
\hline $112^{*}$ & $\mathrm{I}+\mathrm{NO}_{2}^{-} \rightarrow \mathrm{NO}_{2}+\mathrm{I}^{-}$ & $2.5 \times 10^{8}$ & 39 \\
\hline $113^{* a}$ & $\mathrm{I}^{-}+\mathrm{HOI}+\mathrm{H}^{+} \rightarrow \mathrm{I}_{2}+\mathrm{H}_{2} \mathrm{O}$ & $4.4 \times 10^{12}$ & 19 \\
\hline $114^{*}$ & $\mathrm{I}_{2}+\mathrm{H}_{2} \mathrm{O} \rightarrow \mathrm{HOI}+\mathrm{I}^{-}+\mathrm{H}^{+}$ & 3.0 & 19 \\
\hline $115^{*}$ & $\mathrm{I}^{-}+\mathrm{I}_{2} \rightarrow \mathrm{I}_{3}^{-}$ & $5.6 \times 10^{9}$ & 37 \\
\hline $116^{* \mathrm{~b}}$ & $\mathrm{I}_{3}^{-} \rightarrow \mathrm{I}_{2}+\mathrm{I}^{-}$ & $7.5 \times 10^{6}$ & 37 \\
\hline $117^{*}$ & $\mathrm{I}^{-}+\mathrm{HOI}^{-} \rightarrow \mathrm{I}_{2}^{-}+\mathrm{OH}^{-}$ & $6.5 \times 10^{8}$ & 38 \\
\hline $118^{*}$ & $\mathrm{I}_{2}^{-}+\mathrm{OH}^{-} \rightarrow \mathrm{HOI}^{-}+\mathrm{I}^{-}$ & $2.8 \times 10^{4}$ & 38 \\
\hline $119^{*}$ & $\mathrm{I}^{-}+\mathrm{I}_{3}^{-} \rightarrow 2 \mathrm{I}_{2}^{-}$ & $1.25 \times 10^{5}$ & 40 \\
\hline $120^{*}$ & $2 \mathrm{I}_{2}{ }^{-} \rightarrow \mathrm{I}^{-}{ }^{-}+\mathrm{I}^{-}$ & $1.5 \times 10^{9}$ & 40 \\
\hline $121 *$ & $\mathrm{I}+\mathrm{OH}^{-} \rightarrow \mathrm{HOI}^{-}$ & $1.6 \times 10^{8}$ & 38 \\
\hline $122 * \mathrm{~b}$ & $\mathrm{HOI}^{-} \rightarrow \mathrm{I}+\mathrm{OH}^{-}$ & $1.2 \times 10^{8}$ & 37 \\
\hline $123 *$ & $\mathrm{I}+\mathrm{I} \rightarrow \mathrm{I}_{2}$ & $1.5 \times 10^{10}$ & 37 \\
\hline $124 *$ & $\mathrm{I}+\mathrm{I}_{2}^{-} \rightarrow \mathrm{I}_{3}^{-}$ & $4.6 \times 10^{9}$ & 37 \\
\hline $125^{*}$ & $\mathrm{I}+\mathrm{HSO}_{3}^{-} \rightarrow \mathrm{I}^{-}+\mathrm{H}^{+}+\mathrm{SO}_{3}^{-}$ & $1.0 \times 10^{9}$ & 41 \\
\hline
\end{tabular}




\begin{tabular}{|c|c|c|c|}
\hline $126^{*}$ & $\mathrm{I}_{2}^{-}+\mathrm{NO}_{2} \rightarrow \mathrm{I}_{2}+\mathrm{NO}_{2}^{-}$ & $3.25 \times 10^{9}$ & 39 \\
\hline $127^{*}$ & $\mathrm{I}_{2}^{-}+\mathrm{NO}_{2}^{-} \rightarrow 2 \mathrm{I}^{-}+\mathrm{NO}_{2}$ & $3.0 \times 10^{7}$ & 39 \\
\hline $128^{*}$ & $\mathrm{I}_{2}^{-}+\mathrm{HSO}_{3}^{-} \rightarrow 2 \mathrm{I}^{-}+\mathrm{H}^{+}+\mathrm{SO}_{3}^{-}$ & $1.1 \times 10^{6}$ & 34 \\
\hline $129^{*}$ & $\mathrm{I}_{2}^{-}+\mathrm{HOI}^{-} \rightarrow \mathrm{I}_{3}^{-}+\mathrm{OH}^{-}$ & $1.8 \times 10^{10}$ & 38 \\
\hline $130^{*}$ & $\mathrm{I}_{2}+\mathrm{OH} \rightarrow \mathrm{I}+\mathrm{HOI}$ & $9.5 \times 10^{9}$ & 37 \\
\hline $131 * \mathrm{~b}$ & $\begin{array}{c}\mathrm{I}_{2}+\mathrm{HSO}_{3}^{-} \rightarrow \mathrm{ISO}_{3}^{-}+\mathrm{I}^{-}+\mathrm{H}^{+} \\
\mathrm{k}_{131}\left[\mathrm{I}_{2}\right]^{\mathrm{c}}\end{array}$ & $\begin{array}{l}\mathrm{k}_{131} \approx 30.0 \\
(\text { assumed })\end{array}$ & $\begin{array}{c}\text { This } \\
\text { study }^{42}\end{array}$ \\
\hline $132^{*}$ & $\mathrm{HOI}+\mathrm{I}_{2}^{-} \rightarrow \mathrm{I}^{-}+\mathrm{I}^{-}+\mathrm{IO}+\mathrm{H}^{+}$ & $\sim 1.0 \times 10^{5}$ & 43 \\
\hline $133^{*}$ & $\begin{array}{c}\mathrm{HOI}+\mathrm{HSO}_{3}^{-} \rightarrow \mathrm{I}^{-}+\mathrm{SO}_{4}{ }^{2-}+2 \mathrm{H}^{+} \\
\mathrm{k}_{133}[\mathrm{HOI}]\left[\mathrm{HSO}_{3}{ }^{-}\right]^{\mathrm{c}}\end{array}$ & $\mathrm{k}_{133}=5.0 \times 10^{9}$ & 44 \\
\hline $134 * \mathrm{~b}$ & $\begin{array}{c}\mathrm{I}_{3}{ }^{-}+\mathrm{HSO}_{3}{ }^{-} \rightarrow \mathrm{ISO}_{3}{ }^{-}+2 \mathrm{I}^{-}+\mathrm{H}^{+} \\
\mathrm{k}_{134}\left[\mathrm{I}_{2}\right]^{\mathrm{c}}\end{array}$ & $\begin{array}{l}\mathrm{k}_{134} \approx 30.0 \\
(\text { assumed })\end{array}$ & $\begin{array}{c}\text { This } \\
\text { study }^{42}\end{array}$ \\
\hline $135^{* \mathrm{~b}}$ & $\mathrm{I}_{3}^{-}+h v \rightarrow \mathrm{I}_{2}^{-}+\mathrm{I}$ & $1.97 \times 10^{-2}$ & $\begin{array}{c}\text { This } \\
\text { study }{ }^{4,45,46}\end{array}$ \\
\hline $136^{* \mathrm{~b}}$ & $\begin{array}{c}\mathrm{ISO}_{3}^{-}+\mathrm{H}_{2} \mathrm{O} \rightarrow \mathrm{I}^{-}+\mathrm{SO}_{4}{ }^{2-}+2 \mathrm{H}^{+} \\
\mathrm{k}_{136}\left[\mathrm{ISO}_{3}\right]^{-}\end{array}$ & $\mathrm{k}_{136}=2.98 \times 10^{2}$ & 42 \\
\hline \multicolumn{4}{|c|}{ Oxalic acid mechanisms } \\
\hline 137 & $\mathrm{Oxlac}^{-}+\mathrm{OH} \rightarrow \mathrm{CO}_{2}+\mathrm{CO}_{2}^{-}+2 \mathrm{H}_{2} \mathrm{O}$ & $4.7 \times 10^{7}$ & 9 \\
\hline 138 & $\mathrm{Oxlac}+\mathrm{OH} \rightarrow 2 \mathrm{CO}_{2}+2 \mathrm{H}_{2} \mathrm{O}$ & $1.4 \times 10^{6}$ & 9 \\
\hline 139 & $\mathrm{CO}_{2}^{-}+\mathrm{NO} \rightarrow \mathrm{NO}^{-}+\mathrm{CO}_{2}$ & $2.9 \times 10^{9}$ & 33 \\
\hline 140 & $\mathrm{NO}^{-}+\mathrm{NO} \rightarrow \mathrm{N}_{2} \mathrm{O}_{2}^{-}$ & $1.7 \times 10^{9}$ & 47 \\
\hline 141 & $\mathrm{~N}_{2} \mathrm{O}_{2}^{-}+\mathrm{NO} \rightarrow \mathrm{N}_{3} \mathrm{O}_{3}^{-}$ & $3.0 \times 10^{6}$ & 47 \\
\hline 142 & $\mathrm{~N}_{3} \mathrm{O}_{3}^{-} \rightarrow \mathrm{NO}_{2}^{-}+\mathrm{N}_{2} \mathrm{O}$ & 87 & 48 \\
\hline 143 & $\mathrm{SO}_{3}{ }^{-}+\mathrm{N}_{2} \mathrm{O} \rightarrow \mathrm{N}_{2}+\mathrm{SO}_{4}{ }^{2-}+\mathrm{O}^{-}$ & $1.0 \times 10^{4}$ & 49 \\
\hline 144 & $\mathrm{SO}_{2}+\mathrm{CO}_{2}^{-} \rightarrow \mathrm{CO}_{2}+\mathrm{SO}_{2}^{-}$ & $7.6 \times 10^{8}$ & 50 \\
\hline 145 & $\mathrm{CO}_{2}^{-}+\mathrm{O}_{2} \rightarrow \mathrm{O}_{2}^{-}+\mathrm{CO}_{2}$ & $2.4 \times 10^{9}$ & 9 \\
\hline 146 & $\mathrm{O}_{3}+\mathrm{O}_{2}^{-} \rightarrow \mathrm{O}_{2}+\mathrm{O}_{3}^{-}$ & $1.55 \times 10^{9}$ & 12 \\
\hline 147 & $\mathrm{NO}_{3}+\mathrm{O}_{2}^{-} \rightarrow \mathrm{NO}_{3}^{-}+\mathrm{O}_{2}$ & $1.0 \times 10^{9}$ & 7 \\
\hline 148 & $\mathrm{O}_{3}{ }^{-}+\mathrm{H}^{+} \rightarrow \mathrm{OH}+\mathrm{O}_{2}$ & $9.0 \times 10^{9}$ & 18 \\
\hline 149 & $\mathrm{OH}+\mathrm{O}_{2}^{-} \rightarrow \mathrm{OH}^{-}+\mathrm{O}_{2}$ & $1.0 \times 10^{10}$ & 9 \\
\hline
\end{tabular}




\begin{tabular}{|c|c|c|c|}
\hline 150 & $2 \mathrm{O}_{2}^{-}+2 \mathrm{H}_{2} \mathrm{O} \rightarrow \mathrm{H}_{2} \mathrm{O}_{2}+\mathrm{O}_{2}+2 \mathrm{OH}^{-}$ & $<0.3$ & 7 \\
\hline 151 & $\mathrm{OH}+\mathrm{H}_{2} \mathrm{O}_{2} \rightarrow \mathrm{H}_{2} \mathrm{O}+\mathrm{HO}_{2}$ & $2.7 \times 10^{7}$ & 7 \\
\hline 152 & $\mathrm{O}_{2}^{-}+\mathrm{H}_{2} \mathrm{O}_{2} \rightarrow \mathrm{OH}+\mathrm{O}_{2}+\mathrm{OH}^{-}$ & 0.13 & 7 \\
\hline 153 & $\mathrm{O}_{2}^{-}+\mathrm{HO}_{2}+\mathrm{H}_{2} \mathrm{O} \rightarrow \mathrm{H}_{2} \mathrm{O}_{2}+\mathrm{O}_{2}+\mathrm{OH}^{-}$ & $1.0 \times 10^{8}$ & 7 \\
\hline 154 & $\mathrm{SO}_{5}{ }^{-}+\mathrm{O}_{2}{ }^{-}+\mathrm{H}_{2} \mathrm{O} \rightarrow \mathrm{HSO}_{5}^{-}+\mathrm{OH}^{-}+\mathrm{O}_{2}$ & $1.0 \times 10^{8}$ & 7 \\
\hline 155 & $\mathrm{SO}_{4}^{-}+\mathrm{O}_{2}^{-} \rightarrow \mathrm{SO}_{4}{ }^{2-}+\mathrm{O}_{2}$ & $5.0 \times 10^{9}$ & 7 \\
\hline 156 & $\mathrm{~S}(\mathrm{IV})+\mathrm{O}_{2}^{-}+\mathrm{H}_{2} \mathrm{O} \rightarrow \mathrm{S}(\mathrm{VI})+\mathrm{OH}+\mathrm{OH}^{-}$ & $1.0 \times 10^{5}$ & 7 \\
\hline \multicolumn{4}{|c|}{$\mathrm{HCO}_{3}{ }^{-}$mechanisms } \\
\hline 157 & $\mathrm{HCO}_{3}{ }^{-}+\mathrm{OH} \rightarrow \mathrm{H}_{2} \mathrm{O}+\mathrm{CO}_{3}^{-}$ & $1.5 \times 10^{7}$ & 7 \\
\hline 158 & $\mathrm{NO}_{2}{ }^{-}+\mathrm{CO}_{3}^{-} \rightarrow \mathrm{NO}_{2}+\mathrm{CO}_{3}{ }^{2-}$ & $4.0 \times 10^{5}$ & 7 \\
\hline 159 & $\mathrm{NO}_{2}+\mathrm{CO}_{3}{ }^{-} \rightarrow \mathrm{NO}_{3}{ }^{-}+\mathrm{CO}_{2}$ & $1.0 \times 10^{9}$ & 51 \\
\hline 160 & $\mathrm{CO}_{3}{ }^{-}+\mathrm{NO} \rightarrow \mathrm{NO}_{2}^{-}+\mathrm{CO}_{2}$ & $3.5 \times 10^{9}$ & 33 \\
\hline 161 & $\mathrm{CO}_{3}{ }^{-}+\mathrm{SO}_{3}{ }^{-} \rightarrow \mathrm{CO}_{2}+\mathrm{SO}_{4}{ }^{2-}$ & $5.5 \times 10^{8}$ & 51 \\
\hline 162 & $\mathrm{CO}_{3}^{2-}+\mathrm{OH} \rightarrow \mathrm{CO}_{3}^{-}+\mathrm{OH}^{-}$ & $3.9 \times 10^{8}$ & 52 \\
\hline 163 & $\mathrm{SO}_{4}^{-}+\mathrm{HCO}_{3}^{-} \rightarrow \mathrm{SO}_{4}^{2-}+\mathrm{H}^{+}+\mathrm{CO}_{3}^{-}$ & $9.1 \times 10^{6}$ & 7 \\
\hline 164 & $\begin{array}{c}2 \mathrm{HCO}_{3}{ }^{-}+\mathrm{S}(\mathrm{IV})+0.5 \mathrm{O}_{2} \rightarrow \mathrm{SO}_{4}{ }^{2-}+2 \mathrm{CO}_{2}+ \\
\mathrm{H}_{2} \mathrm{O}\end{array}$ & $2.25 \times 10^{6}$ & 8 \\
\hline
\end{tabular}

* The reactions are used in the kinetic model.

a) The units are $\mathrm{M}^{-2} \mathrm{~s}^{-1}$.

b) The units are $\mathrm{s}^{-1}$.

c) The expressions are used to calculate sulfate production rates in the kinetic model except Reaction 47. 
Table S2. Initial concentration of $\mathrm{X}^{-}\left(\mathrm{X}^{-}=\mathrm{Cl}^{-}, \mathrm{Br}^{-}\right.$, and $\left.\mathrm{I}^{-}\right)$and $\mathrm{NO}_{3}{ }^{-}$at $\left[\mathrm{X}^{-}\right]_{0} /\left[\mathrm{NO}_{3}{ }^{-}\right]_{0}$ of 0.2 in $\mathrm{N}_{2}$.

\begin{tabular}{|c|c|c|}
\hline Particle Composition & {$\left[\mathrm{NO}_{3}{ }^{-}\right]_{0}(\mathrm{M})$} & {$\left[\mathrm{Cl}^{-}\right]_{0}$ or $\left[\mathrm{Br}^{-}\right]_{0}$ or $\left[\mathrm{I}^{-}\right]_{0}(\mathrm{M})$} \\
\hline $\mathrm{NH}_{4} \mathrm{NO}_{3}{ }^{\mathrm{a}}$ & 6.6 & N/A \\
\hline $\mathrm{NH}_{4} \mathrm{NO}_{3}+\mathrm{NH}_{4} \mathrm{Cl}^{\mathrm{a}}$ & 5.24 & 1.05 \\
\hline $\mathrm{NH}_{4} \mathrm{NO}_{3}+\mathrm{NH}_{4} \mathrm{Br}^{\mathrm{b}}$ & 5.91 & 1.18 \\
\hline $\mathrm{NH}_{4} \mathrm{NO}_{3}+\mathrm{NH}_{4} \mathrm{I}^{\mathrm{b}}$ & 5.64 & 1.13 \\
\hline
\end{tabular}

a) The initial concentration was calculated based on the E-AIM model.

b) The initial concentration of $\mathrm{NO}_{3}{ }^{-}$was estimated based on the calibration curve of Raman spectra of standard $\mathrm{NH}_{4} \mathrm{NO}_{3}$ solution at different concentrations (Figure S5), and those of $\mathrm{Br}^{-}$and $\mathrm{I}^{-}$were calculated using the initial molar ratio of 0.2 .

Table S3. Reactive uptake coefficients of $\mathrm{SO}_{2}$ for sulfate production in the presence of halide ions and $\mathrm{OH}$ scavengers.

\begin{tabular}{|c|c|c|c|}
\hline \multicolumn{2}{|c|}{ System } & Air condition & $\mathrm{N}_{2}$ condition \\
\hline \multicolumn{2}{|c|}{$\mathrm{NH}_{4} \mathrm{NO}_{3}$} & $(1.87 \pm 0.09) \times 10^{-5}$ & $(2.18 \pm 0.15) \times 10^{-5}$ \\
\hline \multirow{7}{*}{$\begin{array}{c}\mathrm{NH}_{4} \mathrm{NO}_{3}+ \\
\mathrm{NH}_{4} \mathrm{Cl}^{\mathrm{a}}\end{array}$} & 0.05 & $(1.90 \pm 0.23) \times 10^{-5}$ & N/A \\
\hline & 0.1 & $(2.43 \pm 0.25) \times 10^{-5}$ & $(2.14 \pm 0.26) \times 10^{-5}$ \\
\hline & 0.2 & $(2.71 \pm 0.19) \times 10^{-5}$ & $(2.83 \pm 0.22) \times 10^{-5}$ \\
\hline & 0.4 & $(2.02 \pm 0.17) \times 10^{-5}$ & N/A \\
\hline & 1 & $(1.99 \pm 0.13) \times 10^{-5}$ & $(1.11 \pm 0.11) \times 10^{-5}$ \\
\hline & 3 & $(1.33 \pm 0.08) \times 10^{-5}$ & $(0.54 \pm 0.06) \times 10^{-5}$ \\
\hline & 9 & $(0.61 \pm 0.04) \times 10^{-5}$ & $(0.20 \pm 0.02) \times 10^{-5}$ \\
\hline \multicolumn{2}{|c|}{$\mathrm{NH}_{4} \mathrm{NO}_{3}+\mathrm{NH}_{4} \mathrm{Br}^{\mathrm{b}}$} & $(2.54 \pm 0.24) \times 10^{-5}$ & $(2.69 \pm 0.33) \times 10^{-5}$ \\
\hline \multicolumn{2}{|c|}{$\mathrm{NH}_{4} \mathrm{NO}_{3}+\mathrm{NH}_{4} \mathrm{I}^{\mathrm{b}}$} & $(3.27 \pm 0.43) \times 10^{-5}$ & $(4.31 \pm 0.52) \times 10^{-5}$ \\
\hline \multicolumn{2}{|c|}{$\mathrm{NH}_{4} \mathrm{NO}_{3}+\mathrm{NaHCO}_{3}{ }^{\mathrm{b}}$} & $(2.14 \pm 0.13) \times 10^{-5}$ & $(1.89 \pm 0.27) \times 10^{-5}$ \\
\hline \multicolumn{2}{|c|}{$\mathrm{NH}_{4} \mathrm{NO}_{3}+\mathrm{Oxlac}^{\mathrm{b}}$} & $(2.13 \pm 0.35) \times 10^{-5}$ & $(2.18 \pm 0.30) \times 10^{-5}$ \\
\hline
\end{tabular}

a) At different initial molar ratio of $\mathrm{Cl}^{-}$to $\mathrm{NO}_{3}{ }^{-}$.

b) At $\left[\mathrm{Br}^{-}\right]_{0} /\left[\mathrm{NO}_{3}{ }^{-}\right]_{0},\left[\mathrm{I}^{-}\right]_{0} /\left[\mathrm{NO}_{3}{ }^{-}\right]_{0},\left[\mathrm{HCO}_{3}{ }^{-}\right]_{0} /\left[\mathrm{NO}_{3}{ }^{-}\right]_{0}$, and $[\mathrm{Oxlac}]_{0} /\left[\mathrm{NO}_{3}{ }^{-}\right]_{0}$ of 0.2 . 
Table S4. Equilibrium constants or mass transport coefficients (Values are given for 298.15 K).

\begin{tabular}{|c|c|c|}
\hline & Constant or coefficient & Reference \\
\hline $\mathrm{R}_{\mathrm{p}}$ & $26.0 \mu \mathrm{m}$ & This study \\
\hline $\mathrm{k}_{\mathrm{f}}$ & $3.4 \times 10^{6} \mathrm{~s}^{-1}$ & 6,7 \\
\hline $\mathrm{k}_{\mathrm{r}}$ & $2 \times 10^{8} \mathrm{M}^{-1} \cdot \mathrm{s}^{-1}$ & 6,7 \\
\hline $\mathrm{D}_{\mathrm{g}}$ & $1.089 \times 10^{-5} \mathrm{~m}^{2} \mathrm{~s}^{-1}$ & 53 \\
\hline $\mathrm{D}_{\mathrm{aq}}$ & $1.32 \times 10^{-9} \mathrm{~m}^{2} \mathrm{~s}^{-1}$ & 54 \\
\hline$\alpha \mathrm{so2}^{*}$ & 0.11 & 7 \\
\hline $\mathrm{HsO}^{*}$ & $1.23 \mathrm{M} \mathrm{atm}^{-1}$ & 7 \\
\hline
\end{tabular}




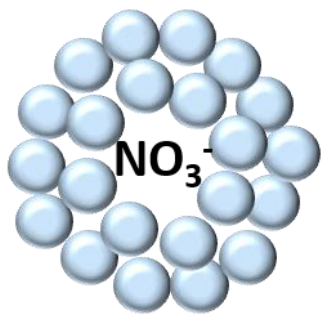

In bulk phase

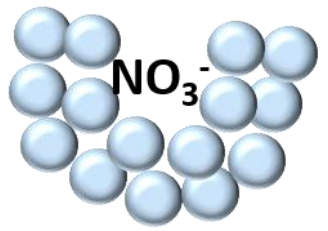

At interface

\section{water molecule}

Figure S1. Complete solvent cage in bulk phase and incomplete solvent cage at air-particle interface.

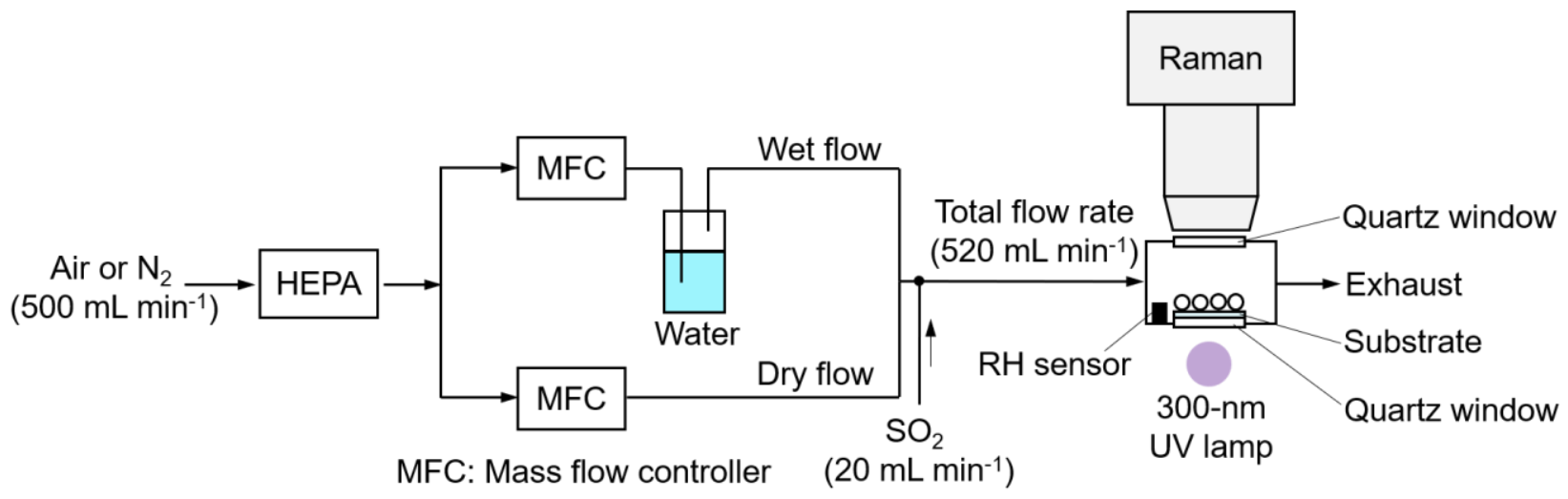

Figure S2. Schematic of experimental setup. 


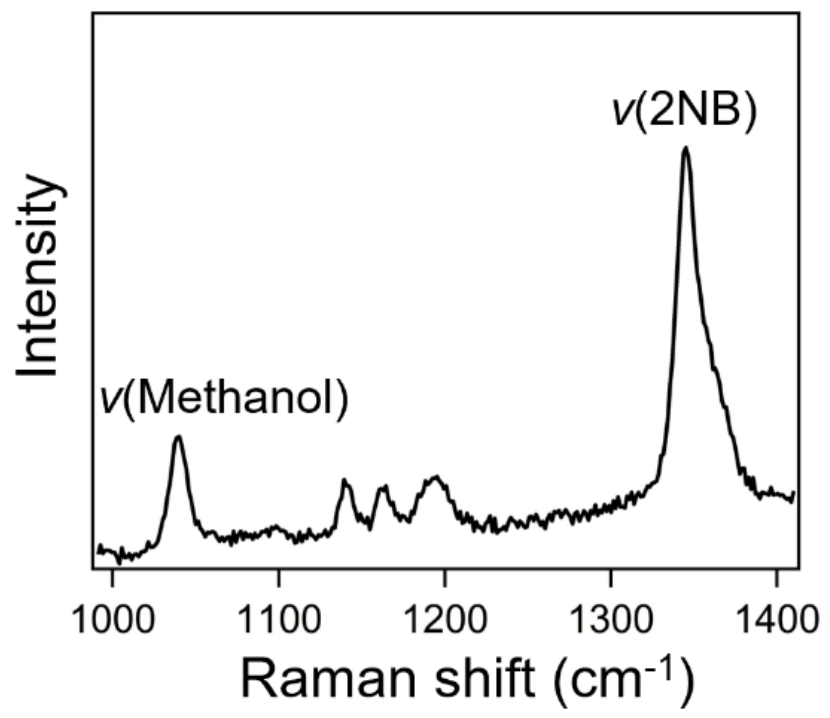

Figure S3. Raman spectrum of 2NB dissolved into methanol.

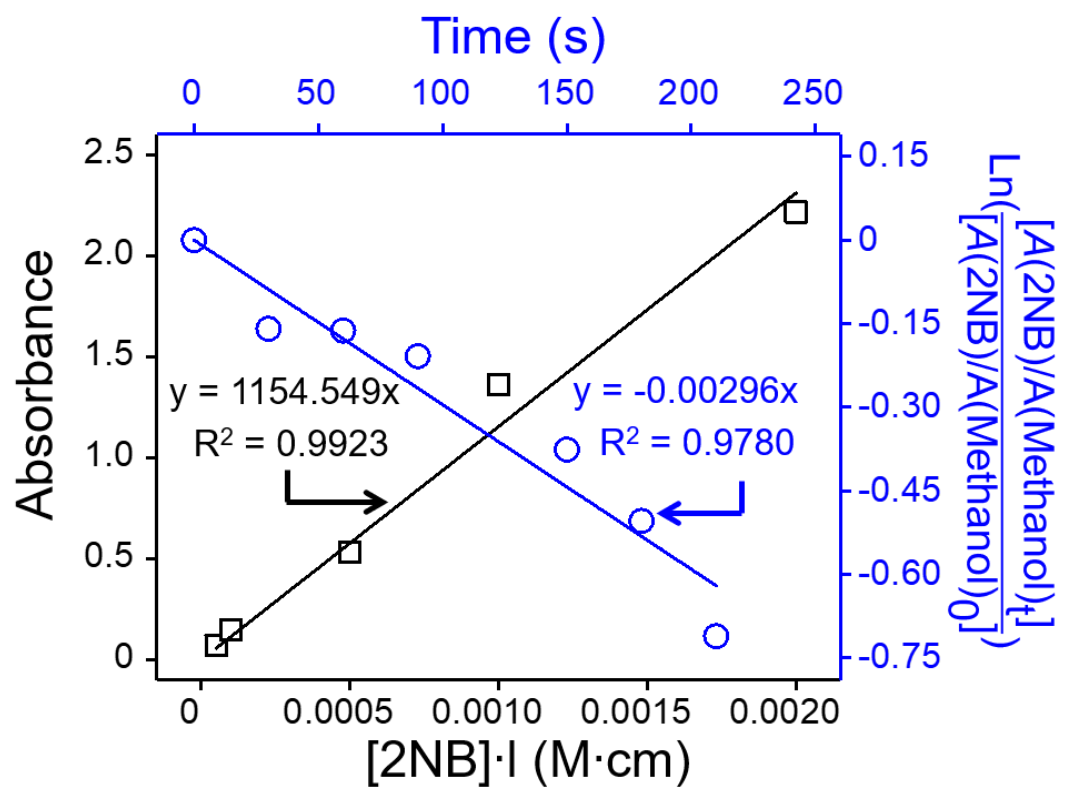

Figure S4. Absorbance of $2 \mathrm{NB}$ as a function of $2 \mathrm{NB}$ concentration and cuvette path length (black). Photodecay plot of $2 \mathrm{NB}$ in particle (blue). Circle $(O)$ and square $(\square)$ represent measured data points; blue and black solid lines are fitted regression lines. 

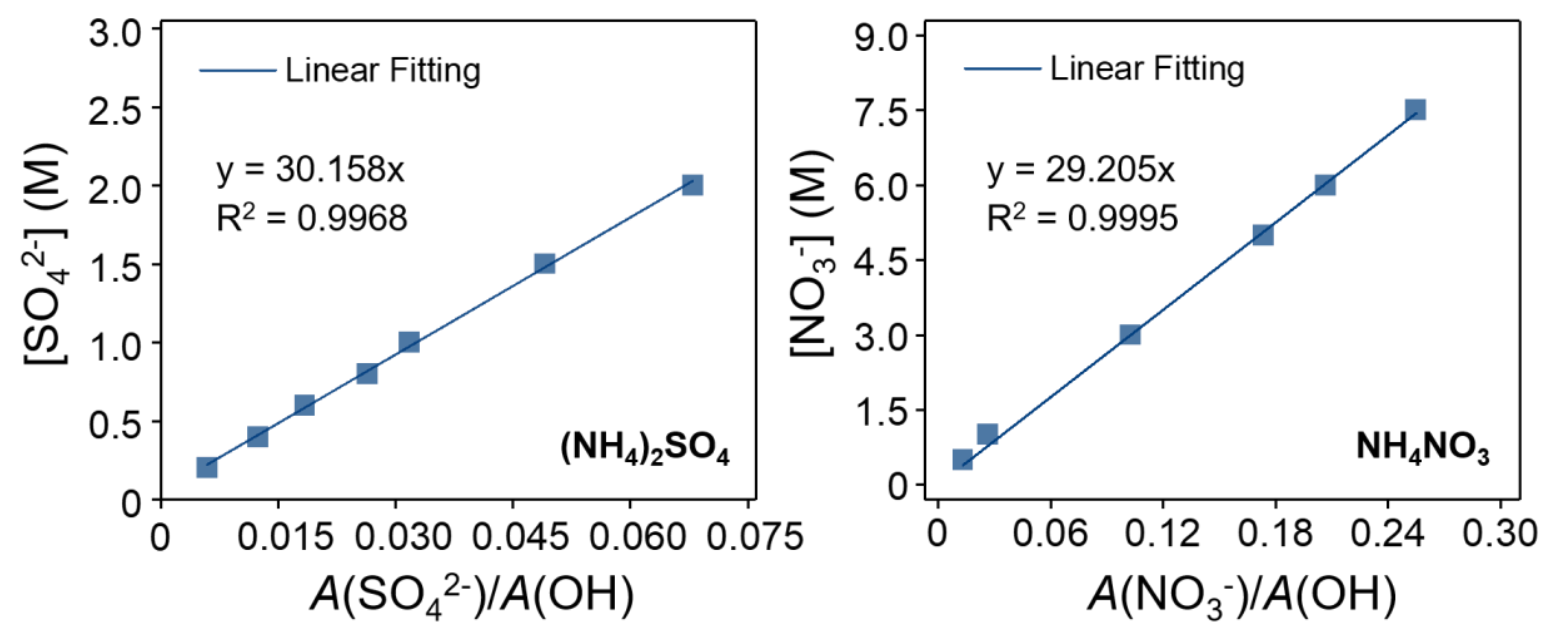

Figure S5. Calibration plot of sulfate concentration and nitrate concentration. $\left[\mathrm{SO}_{4}{ }^{2-}\right]$ in standard ammonium sulfate versus the peak area ratio of $v\left(\mathrm{SO}_{4}{ }^{2-}\right)$ to $v(\mathrm{OH})_{\text {water. }}\left[\mathrm{NO}_{3}{ }^{-}\right]$in standard ammonium nitrate versus the peak area ratio of $v\left(\mathrm{NO}_{3}{ }^{-}\right)$to $v(\mathrm{OH})_{\text {water. }}$

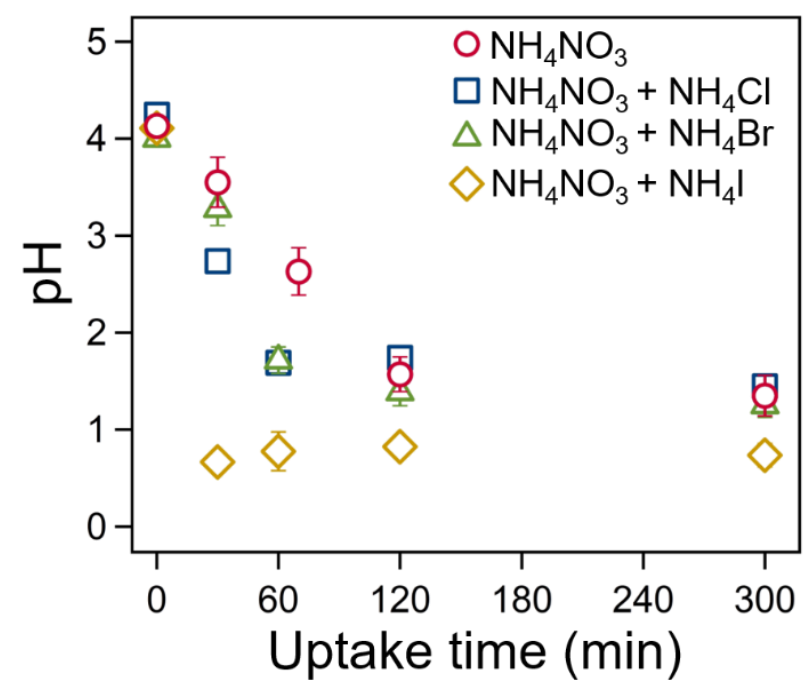

Figure S6. Change in particle $\mathrm{pH}$ during sulfate formation for particles of $\mathrm{NH}_{4} \mathrm{NO}_{3}, \mathrm{NH}_{4} \mathrm{NO}_{3}+$ $\mathrm{NH}_{4} \mathrm{Cl}, \mathrm{NH}_{4} \mathrm{NO}_{3}+\mathrm{NH}_{4} \mathrm{Br}$, and $\mathrm{NH}_{4} \mathrm{NO}_{3}+\mathrm{NH}_{4} \mathrm{I}$ in $\mathrm{N}_{2}$ condition at initial molar ratio of $\mathrm{X}^{-}$to $\mathrm{NO}_{3}{ }^{-}$ of $0.2\left(\mathrm{X}^{-}=\mathrm{Cl}^{-} / \mathrm{Br}^{-} / \mathrm{I}^{-}\right)$. 


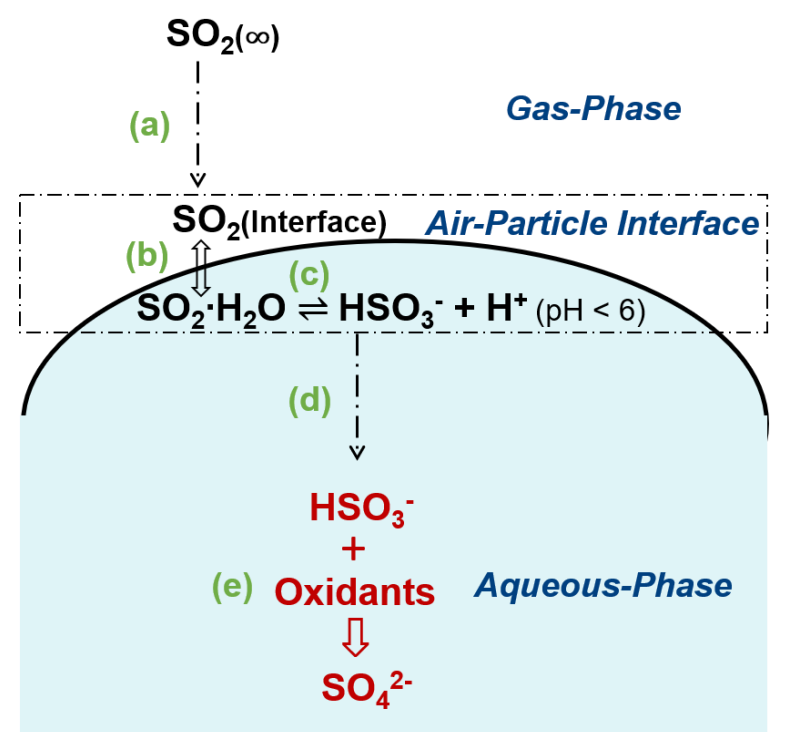

Figure S7. Mass transport of $\mathrm{SO}_{2}$ in gas phase and of dissolved $\mathrm{SO}_{2}$ in aqueous phase. The letters indicate (a) gas phase diffusion, (b) dissolution equilibrium of $\mathrm{SO}_{2}$ at the air-particle interface (Henry's law); (c) hydrolysis of $\mathrm{SO}_{2}$ and partial dissociation to $\mathrm{HSO}_{3}{ }^{-}$; (d) aqueous phase diffusion of $\mathrm{HSO}_{3}^{-}$; (e) aqueous oxidation process of $\mathrm{HSO}_{3}^{-}$.

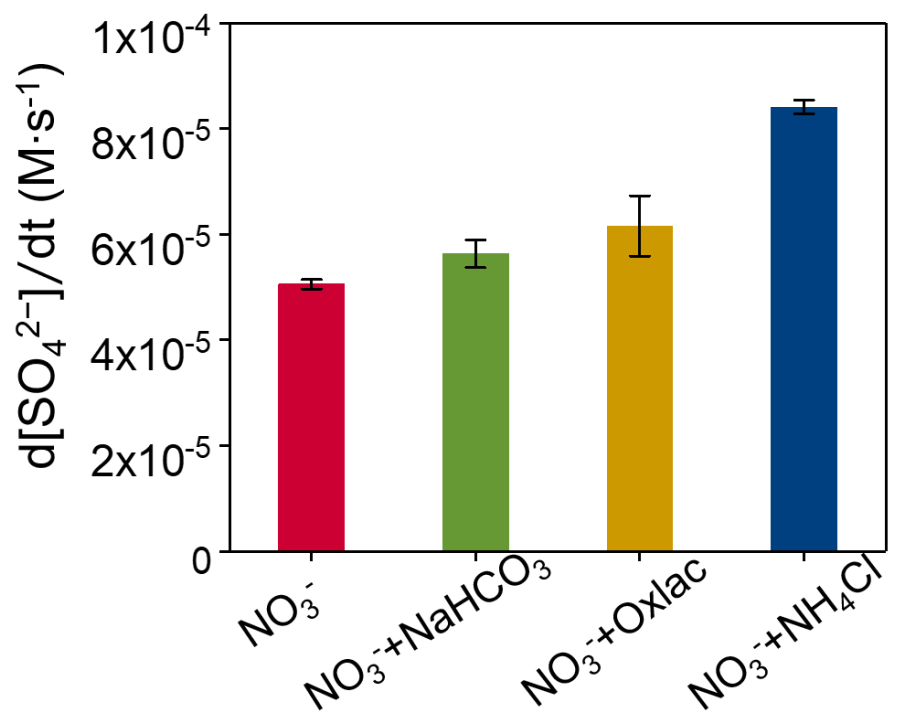

Figure S8. Sulfate production rate of pure $\mathrm{NH}_{4} \mathrm{NO}_{3}$ and mixture of $\mathrm{NH}_{4} \mathrm{NO}_{3}+\mathrm{NaHCO}_{3}, \mathrm{NH}_{4} \mathrm{NO}_{3}$ + Oxlac, and $\mathrm{NH}_{4} \mathrm{NO}_{3}+\mathrm{NH}_{4} \mathrm{Cl}$ in air condition at initial molar ratio of $\mathrm{OH}$ scavenger to nitrate of 0.2 . 

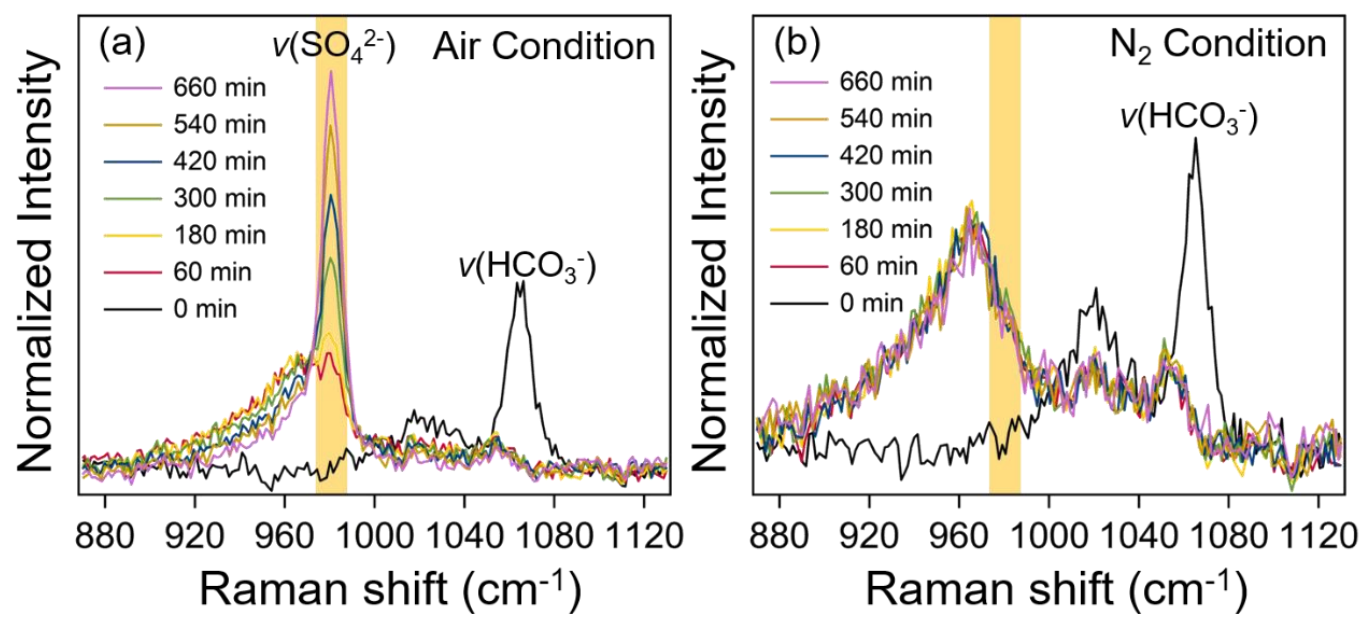

Figure S9. Raman spectra of sodium bicarbonate particles in the presence of $\mathrm{SO}_{2}(7.7 \mathrm{ppm})$ under dark condition in (a) air and (b) $\mathrm{N}_{2}$ condition. The shaded area represents peak of $v\left(\mathrm{SO}_{4}{ }^{2-}\right)$ mode at $\sim 979 \mathrm{~cm}^{-1}$.

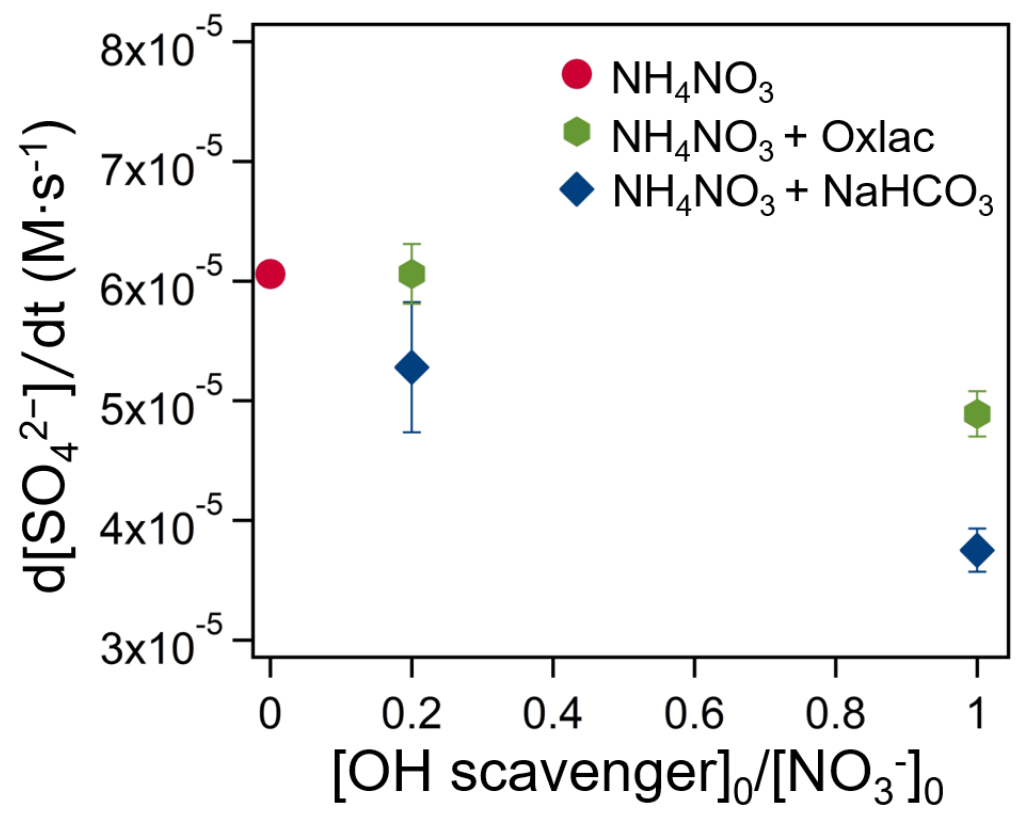

Figure S10. Sulfate production rate under initial molar ratio of $\mathrm{OH}$ scavenger to $\mathrm{NO}_{3}{ }^{-},[\mathrm{OH}$ scavenger $]_{0} /\left[\mathrm{NO}_{3}^{-}\right]_{0}$, of $0,0.2$ and 1 in $\mathrm{N}_{2}$ condition. 


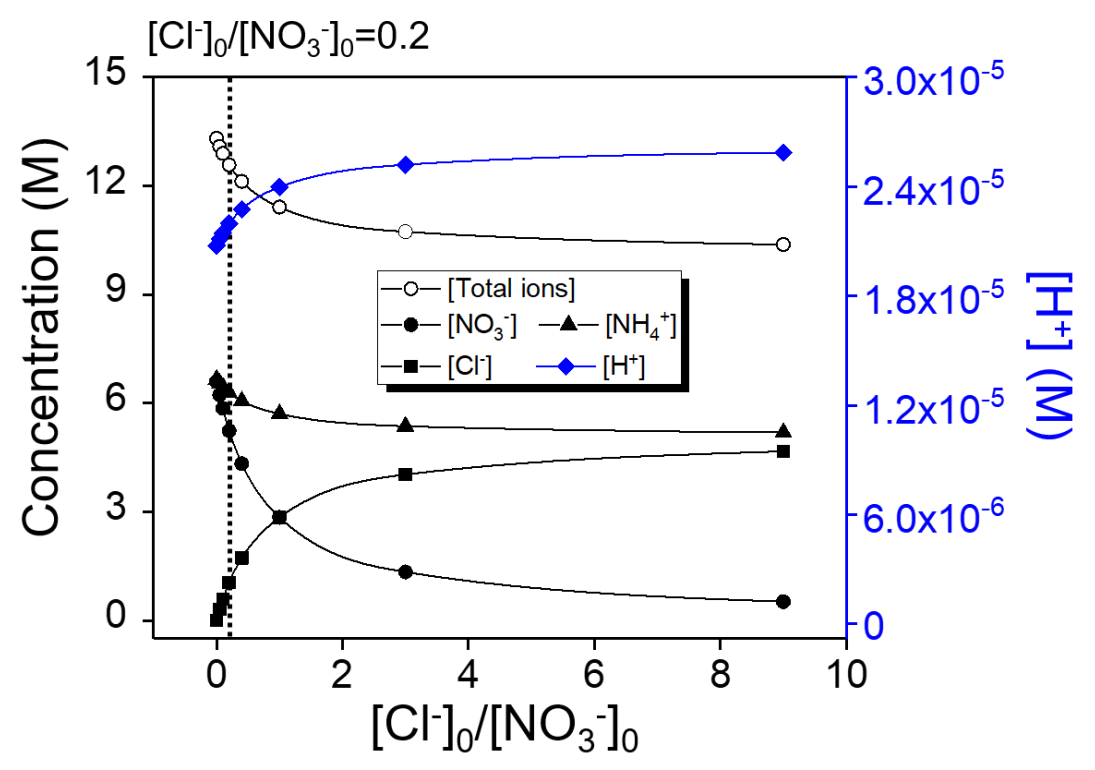

Figure S11. Initial concentration of $\mathrm{NO}_{3}{ }^{-}, \mathrm{Cl}^{-}, \mathrm{NH}_{4}{ }^{+}, \mathrm{H}^{+}$, and total ions at different $\left[\mathrm{Cl}^{-}\right]_{0} /\left[\mathrm{NO}_{3}{ }^{-}\right]_{0}$ based on E-AIM model at $80 \%$ RH.

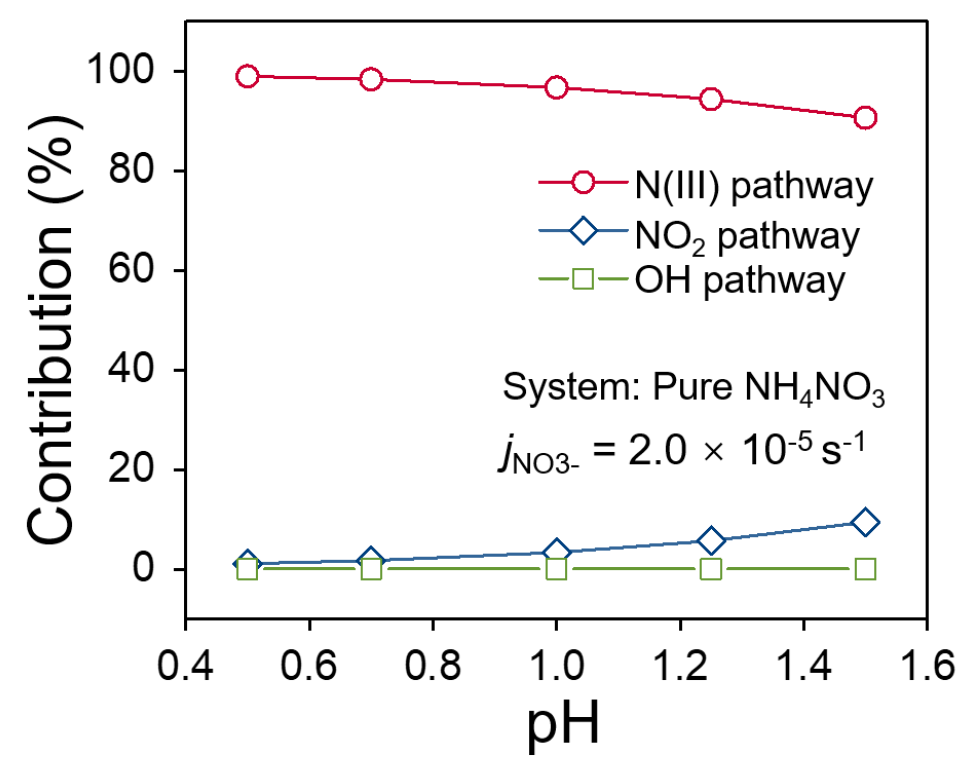

Figure S12. Sensitive test of contribution of $\mathrm{N}(\mathrm{III}), \mathrm{NO}_{2}$, and $\mathrm{OH}$ pathway on sulfate production under different $\mathrm{pH}$ in the kinetic model. (Condition: $j_{\mathrm{NO} 3-}=2.0 \times 10^{-5} \mathrm{~s}^{-1}$ in the only presence of $\mathrm{NO}_{3}{ }^{-}$. 


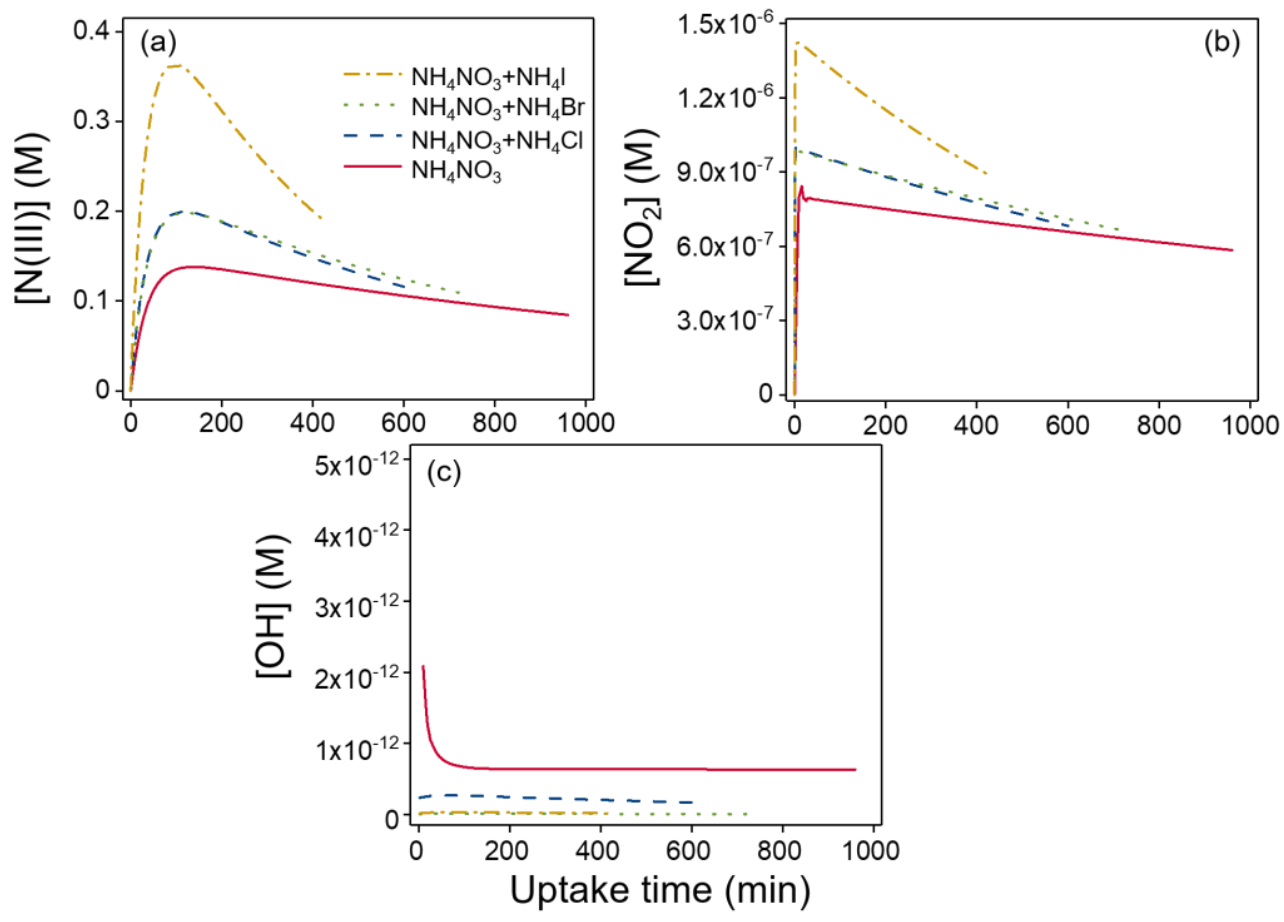

Figure S13. Model results of (a) $\mathrm{N}(\mathrm{III})$, (b) $\mathrm{NO}_{2}$, and (c) $\mathrm{OH}$ radical concentration as a function of uptake time under different chemical systems including pure $\mathrm{NH}_{4} \mathrm{NO}_{3}, \mathrm{NH}_{4} \mathrm{NO}_{3}+\mathrm{NH}_{4} \mathrm{Cl}$, $\mathrm{NH}_{4} \mathrm{NO}_{3}+\mathrm{NH}_{4} \mathrm{Br}$, and $\mathrm{NH}_{4} \mathrm{NO}_{3}+\mathrm{NH}_{4} \mathrm{I}$.

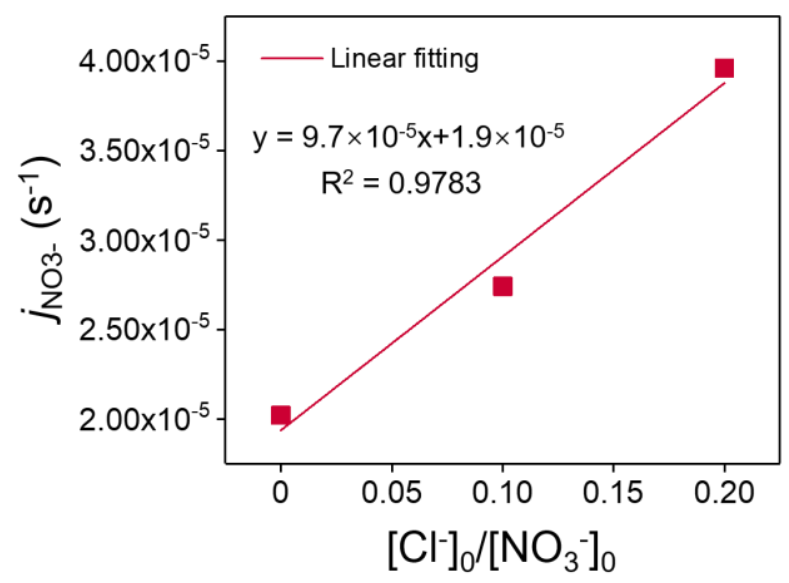

Figure S14. Nitrate photolysis rate constants, $j_{\mathrm{NO} 3}-$, as a function of $\left[\mathrm{Cl}^{-}\right]_{0} /\left[\mathrm{NO}_{3}{ }^{-}\right]_{0}$ under $\mathrm{N}_{2}$ condition. Note that $j_{\mathrm{NO} 3}-$ at $\left[\mathrm{Cl}^{-}\right]_{0} /\left[\mathrm{NO}_{3}{ }^{-}\right]_{0}>0.2$ was not used in the Figure because of the limitation of incorporating the saturation effect of $\mathrm{Cl}^{-}$at the interface in the current kinetic modeling. 


\section{REFERENCE}

(1) Ottosson, N.; Heyda, J.; Wernersson, E.; Pokapanich, W.; Svensson, S.; Winter, B.; Ohrwall, G.; Jungwirth, P.; Bjorneholm, O. The influence of concentration on the molecular surface structure of simple and mixed aqueous electrolytes. Phys. Chem. Chem. Phys. 2010, 12 (36), 10693-10700.

(2) Jungwirth, P.; Tobias, D. J. Molecular Structure of Salt Solutions: A New View of the Interface with Implications for Heterogeneous Atmospheric Chemistry. J. Phys. Chem. B 2001, 105 (43), 10468-10472.

(3) Piatkowski, L.; Zhang, Z.; Backus, E. H.; Bakker, H. J.; Bonn, M. Extreme surface propensity of halide ions in water. Nat. Commun. 2014, 54083.

(4) Galbavy, E. S.; Ram, K.; Anastasio, C. 2-Nitrobenzaldehyde as a chemical actinometer for solution and ice photochemistry. J. Photochem. Photobiol. A: Chem. 2010, 209 (2-3), 186192.

(5) Gardner, J. A.; Watson, L. R.; Adewuyi, Y. G.; Davidovits, P.; Zahniser, M. S.; Worsnop, D. R.; Kolb, C. E. Measurement of the mass accommodation coefficient of $\mathrm{SO}_{2}(\mathrm{~g})$ on water droplets. J. Geophys. Res.: Atmos. 1987, 92 (D9), 10887-10895.

(6) Schwartz, S.; Freiberg, J. Mass-transport limitation to the rate of reaction of gases in liquid droplets: application to oxidation of $\mathrm{SO}_{2}$ in aqueous solutions. Atmospheric Environment (1967) 1981, 15 (7), 1129-1144.

(7) Seinfeld, J. H.; Pandis, S. N. Atmospheric Chemistry and Physics: From Air Pollution to Climate Change; Wiley: New York, 2006.

(8) Keener, T. C.; Khang, S.-J. Kinetics of the sodium bicarbonate-sulfur dioxide reaction. Chem. Eng. Sci. 1993, 48 (16), 2859-2865. 
(9) Tan, Y.; Perri, M. J.; Seitzinger, S. P.; Turpin, B. J. Effects of precursor concentration and acidic sulfate in aqueous glyoxal-OH radical oxidation and implications for secondary organic aerosol. Environ. Sci. Technol. 2009, 43 (21), 8105-8112.

(10) Gen, M.; Zhang, R.; Huang, D. D.; Li, Y.; Chan, C. K. Heterogeneous Oxidation of $\mathrm{SO}_{2}$ in Sulfate Production during Nitrate Photolysis at $300 \mathrm{~nm}$ : Effect of pH, Relative Humidity, Irradiation Intensity, and the Presence of Organic Compounds. Environ. Sci. Technol. 2019, 53 (15), 8757-8766.

(11) Exner, M.; Herrmann, H.; Zellner, R. Laser-based studies of reactions of the nitrate radical in aqueous solution. Berichte der Bunsengesellschaft für physikalische Chemie 1992, 96 (3), 470-477.

(12) Bulman, D. M.; Mezyk, S. P.; Remucal, C. K. The Impact of pH and Irradiation Wavelength on the Production of Reactive Oxidants during Chlorine Photolysis. Environ. Sci. Technol. 2019, 53 (8), 4450-4459.

(13) Loegager, T.; Sehested, K. Formation and decay of peroxynitrous acid: a pulse radiolysis study. J. Phys. Chem. 1993, 97 (25), 6664-6669.

(14) Huie, R. E. The reaction kinetics of $\mathrm{NO}_{2}$. Toxicology 1994, 89 (3), 193-216.

(15) Poskrebyshev, G.; Huie, R. E.; Neta, P. The Rate and Equilibrium Constants for the Reaction $\mathrm{NO}_{3} \bullet+\mathrm{Cl}^{-} \rightleftarrows \mathrm{NO}_{3}{ }^{-}+\mathrm{Cl} \bullet$ in Aqueous Solutions. J. Phys. Chem. A 2003, 107 (12), 1964-1970.

(16) Daniels, M. Radiation chemistry of the aqueous nitrate system. III. Pulse electron radiolysis of concentrated sodium nitrate solutions. J. Phys. Chem. 1969, 73 (11), 3710-3717. 
(17) Oblath, S. B.; Markowitz, S. S.; Novakov, T.; Chang, S. G. Kinetics of the formation of hydroxylamine disulfonate by reaction of nitrite with sulfites. J. Phys. Chem. 1981, 85 (8), 1017-1021.

(18) Sehested, K.; Corfitzen, H.; Holcman, J.; Hart, E. J. On the Mechanism of the Decomposition of Acidic $\mathrm{O}_{3}$ Solutions, Thermally or $\mathrm{H}_{2} \mathrm{O}_{2}$-Initiated. J. Phys. Chem. A 1998, 102 (16), 2667-2672.

(19) Eigen, M.; Kustin, K. The kinetics of halogen hydrolysis. JACS 1962, 84 (8), 1355-1361.

(20) Guo, K.; Wu, Z.; Shang, C.; Yao, B.; Hou, S.; Yang, X.; Song, W.; Fang, J. Radical Chemistry and Structural Relationships of PPCP Degradation by UV/Chlorine Treatment in Simulated Drinking Water. Environ. Sci. Technol. 2017, 51 (18), 10431-10439.

(21) Huie, R. E.; Clifton, C. L.; Neta, P. Electron transfer reaction rates and equilibria of the carbonate and sulfate radical anions. International Journal of Radiation Applications and Instrumentation. Part C. Radiation Physics and Chemistry 1991, 38 (5), 477-481.

(22) Kläning, U. K.; Wolff, T. Laser Flash Photolysis of $\mathrm{HClO}, \mathrm{ClO}^{-}, \mathrm{HBrO}$, and $\mathrm{BrO}^{-}$in Aqueous Solution. Reactions of $\mathrm{Cl}$ - and Br-Atoms. Berichte der Bunsengesellschaft für physikalische Chemie 1985, 89 (3), 243-245.

(23) Yu, X.-Y.; Barker, J. R. Hydrogen Peroxide Photolysis in Acidic Aqueous Solutions Containing Chloride Ions. I. Chemical Mechanism. J. Phys. Chem. A 2003, 107 (9), 13131324.

(24) Huie, R. E.; Neta, P. Rate constants for some oxidations of S(IV) by radicals in aqueous solutions. Atmos. Environ. 1987, 21 (8), 1743-1747. 
(25) Sun, P.; Lee, W. N.; Zhang, R.; Huang, C. H. Degradation of DEET and Caffeine under UV/Chlorine and Simulated Sunlight/Chlorine Conditions. Environ. Sci. Technol. 2016, 50 (24), 13265-13273.

(26) Horváth, A. K.; Nagypál, I.; Epstein, I. R. Three Autocatalysts and Self-Inhibition in a Single Reaction: A Detailed Mechanism of the Chlorite-Tetrathionate Reaction. Inorg. Chem. 2006, 45 (24), 9877-9883.

(27) Zehavi, D.; Rabani, J. Oxidation of aqueous bromide ions by hydroxyl radicals. Pulse radiolytic investigation. J. Phys. Chem. 1972, 76 (3), 312-319.

(28) Merenyi, G.; Lind, J. Reaction mechanism of hydrogen abstraction by the bromine atom in water. JACS 1994, 116 (17), 7872-7876.

(29) Ershov, B. G. Kinetics, mechanism and intermediates of some radiation-induced reactions in aqueous solutions. Russian chemical reviews 2004, 73 (1), 101-113.

(30) Lu, J.; Wu, J.; Ji, Y.; Kong, D. Transformation of bromide in thermo activated persulfate oxidation processes. Water Res. 2015, 78 1-8.

(31) von Gunten, U.; Hoigne, J. Bromate Formation during Ozonization of Bromide-Containing Waters: Interaction of Ozone and Hydroxyl Radical Reactions. Environ. Sci. Technol. 1994, 28 (7), 1234-1242.

(32) Huie, R. E.; Neta, P. Kinetics of one-electron transfer reactions involving chlorine dioxide and nitrogen dioxide. J. Phys. Chem. 1986, 90 (6), 1193-1198.

(33) Czapski, G.; Holcman, J.; Bielski, B. H. J. Reactivity of Nitric Oxide with Simple ShortLived Radicals in Aqueous Solutions. JACS 1994, 116 (25), 11465-11469.

(34) Neta, P.; Huie, R. E. One-electron redox reactions involving sulfite ions and aromatic amines. J. Phys. Chem. 1985, 89 (9), 1783-1787. 
(35) Chen, Q.; Schmidt, J. A.; Shah, V.; Jaeglé, L.; Sherwen, T.; Alexander, B. Sulfate production by reactive bromine: Implications for the global sulfur and reactive bromine budgets. Geophysical Research Letters 2017, 44 (13), 7069-7078.

(36) Watanabe, K.; Matsuda, S.; Cuevas, C. A.; Saiz-Lopez, A.; Yabushita, A.; Nakano, Y. Experimental Determination of the Photooxidation of Aqueous $\mathrm{I}^{-}$as a Source of Atmospheric I2. ACS Earth Space Chem. 2019, 3 (4), 669-679.

(37) Elliot, A. J. A pulse radiolysis study of the reaction of OH with $\mathrm{I}_{2}$ and the decay of $\mathrm{I}_{2}{ }^{-}$. Can. J. Chem. 1992, 70 (6), 1658-1661.

(38) Mulazzani, Q. G.; Buxton, G. V. On the kinetics and mechanism of the oxidation of $\mathrm{I}^{-}$by $\mathrm{OH} / \mathrm{O}^{-}$in alkaline aqueous solution. Chem. Phys. Lett. 2006, 421 (1-3), 261-265.

(39) Cripps, R. C.; Jäckel, B.; Güntay, S. On the radiolysis of iodide, nitrate and nitrite ions in aqueous solution: An experimental and modelling study. Nucl. Eng. Des. 2011, 241 (8), 3333-3347.

(40) Troian-Gautier, L.; Turlington, M. D.; Wehlin, S. A. M.; Maurer, A. B.; Brady, M. D.; Swords, W. B.; Meyer, G. J. Halide Photoredox Chemistry. Chem. Rev. 2019, 119 (7), 4628-4683.

(41) Alfassi, Z.; Huie, R.; Marguet, S.; Natarajan, E.; Neta, P. Rate constants for reactions of iodine atoms in solution. Int. J. Chem. Kinet. 1995, 27 (2), 181-188.

(42) Yiin, B. S.; Margerum, D. W. Nonmetal redox kinetics: reactions of iodine and triiodide with sulfite and hydrogen sulfite and the hydrolysis of iodosulfate. Inorg. Chem. 1990, 29 (8), 1559-1564.

(43) Buxton, G. V.; Sellers, R. M. Radiation-induced redox reactions of iodine species in aqueous solution. Formation and characterisation of $\mathrm{I}^{\mathrm{II}}, \mathrm{I}^{\mathrm{IV}}, \mathrm{I}^{\mathrm{VI}}$ and $\mathrm{I}^{\mathrm{VIII}}$, the stability of 
hypoiodous acid and the chemistry of the interconversion of iodide and iodate. Journal of the Chemical Society, Faraday Transactions 1: Physical Chemistry in Condensed Phases 1985, 81 (2), 449-471.

(44) Pechtl, S.; Schmitz, G.; Glasow, R. v. Modelling iodide-iodate speciation in atmospheric aerosol: Contributions of inorganic and organic iodine chemistry. Atmos. Chem. Phys. 2007, 7 (5), 1381-1393.

(45) Jung, S.-H.; Yeon, J.-W.; Kang, Y.; Song, K. Determination of Triiodide Ion Concentration Using UV-Visible Spectrophotometry. Asian J. Chem. 2014, 26 (13), 4084-4086.

(46) Goldstein, S.; Rabani, J. The ferrioxalate and iodide-iodate actinometers in the UV region. J. Photochem. Photobiol. A: Chem. 2008, 193 (1), 50-55.

(47) Seddon, W.; Fletcher, J.; Sopchyshyn, F. Pulse radiolysis of nitric oxide in aqueous solution. Can. J. Chem. 1973, 51 (7), 1123-1130.

(48) Grätzel, M.; Taniguchi, S.; Henglein, A. Pulsradiolytische untersuchung kurzlebiger zwischenprodukte der NO-reduktion in wässriger lösung. Berichte der Bunsengesellschaft für physikalische Chemie 1970, 74 (10), 1003-1010.

(49) Sozurakov, D. M. The Branching Ratio of $\mathrm{SO}_{5}^{-}$Radicals Self-Reaction in Aqueous Solution. J. Phys. Chem. 1993, 97 (41), 10712-10714.

(50) Eriksen, T. Pulse radiolytic investigation of the $\mathrm{SO}_{2}$ radical ion. Radiochemical and Radioanalytical Letters 1975, 22 (1), 33-40.

(51) Lilie, J.; Hanrahan, R. J.; Henglein, A. $\mathrm{O}^{-}$transfer reactions of the carbonate radical anion. Radiation Physics and Chemistry (1977) 1978, 11 (5), 225-227.

(52) Huang, Y.; Kong, M.; Westerman, D.; Xu, E. G.; Coffin, S.; Cochran, K. H.; Liu, Y.; Richardson, S. D.; Schlenk, D.; Dionysiou, D. D. Effects of $\mathrm{HCO}_{3}{ }^{-}$on Degradation of 
Toxic Contaminants of Emerging Concern by UV/NO3 ${ }^{-}$. Environ. Sci. Technol. 2018, 52 (21), 12697-12707.

(53) Massman, W. A review of the molecular diffusivities of $\mathrm{H}_{2} \mathrm{O}, \mathrm{CO}_{2}, \mathrm{CH}_{4}, \mathrm{CO}, \mathrm{O}_{3}, \mathrm{SO}_{2}$, $\mathrm{NH}_{3}, \mathrm{~N}_{2} \mathrm{O}, \mathrm{NO}$, and $\mathrm{NO}_{2}$ in air, $\mathrm{O}_{2}$ and $\mathrm{N}_{2}$ near STP. Atmos. Environ. 1998, 32 (6), 1111 1127.

(54) Leaist, D. G. Diffusion coefficient of aqueous sulfur dioxide at $25^{\circ}$ C. J. Chem. Eng. Data 1984, 29 (3), 281-282. 